\title{
Selfish mitochondria exploit nutrient status across different levels of selection
}

\author{
Bryan L. Gitschlag ${ }^{1}$, Ann T. Tate ${ }^{1}$, \& Maulik R. Patel ${ }^{1,2}$ *
}

${ }^{1}$ Department of Biological Sciences, Vanderbilt University, Nashville, TN, USA

${ }^{2}$ Department of Cell and Developmental Biology, Vanderbilt University School of

Medicine, Nashville, TN, USA

${ }^{*}$ Corresponding author: maulik.r.patel@vanderbilt.edu

\begin{abstract}
Cooperation and cheating are widespread evolutionary strategies. Competition can simultaneously favor cheating within groups and cooperation between groups. Selfish or cheater mitochondrial DNA (mtDNA) mutants proliferate within hosts while being selected against at the level of host fitness. How does environment govern competition between cooperators and cheaters across different selection levels? Focusing on food availability, we address this question using heteroplasmic Caenorhabditis elegans. We show that by promoting germline development, nutrient status provides the niche space for mtDNA variants to compete. However, the within-host advantage of selfish mtDNA requires additional conditions, namely the FoxO transcription factor DAF-16. During food scarcity, DAF-16 mitigates the host fitness cost of the selfish mtDNA. We conclude that food availability, and resilience to food scarcity, govern selfish mtDNA dynamics across the levels of selection. Our study integrates an evolutionary framework with experimentation to identify cellular mechanisms underlying the multilevel selection that characterizes cheater dynamics.
\end{abstract}




\section{Introduction}

Life is generally organized into a hierarchy of cooperative collectives, with multiple genes collectively composing a genome, different genomes giving rise to a eukaryotic cell, cells giving rise to multicellular organisms, and multicellular organisms forming societies (Wilson and Wilson, 2007). New levels of organization in the hierarchy emerge when natural selection favors a division of labor and the loss of conflict between previously autonomous replicators, giving rise to a cohesive unit upon which selection can further act (Michod et al., 2006; West et al., 2015). Cooperation thus facilitates evolutionary transitions toward larger, more complex systems (Fisher and Regenberg, 2019; Gulli et al., 2019; Michod et al., 2006; West et al., 2015). However, because cooperation requires investing in the fitness of others for mutual benefit, it also creates the conditions that enable the emergence of selfish "cheater" entities. By benefiting from the cooperative contributions of others without reciprocating, cheaters experience a fitness advantage over their cooperative counterparts (Dobata et al., 2009; Ghoul et al., 2014; Strassmann et al., 2000). This advantage can break down at higher levels of biological organization, which rely on cooperation at lower levels (de Vargas Roditi et al., 2013; Ozkaya et al., 2017). Hence, natural selection can favor a trait at one level of the biological hierarchy while favoring a competing trait at a different level, a phenomenon known as multilevel selection (de Vargas Roditi et al., 2013; Wilson and Wilson, 2007). Multilevel selection thus provides an explanation for the paradoxical coexistence of selfishness and cooperation in an evolving population. 
Competition over limited resources shapes relative reproductive fitness, and hence Darwinian evolution. By ensuring efficient resource utilization, cooperation can provide an adaptive benefit (Koschwanez et al., 2013; Vanthournout et al., 2016). Accordingly, the availability of resources likely represents an important ecological determinant of the population dynamics between selfish and cooperative entities (Ducasse et al., 2015). Prior studies have indicated that resource scarcity can favor cooperative, group-oriented interactions (Cao et al., 2015; Chisholm and Firtel, 2004; Li and Purugganan, 2011; Pereda et al., 2017), whereas resource abundance can promote selfishness (Chen and Perc, 2014). The link between resource availability and the proliferation of selfish entities can be found at multiple levels of scale, with cancer representing a well-known example. Cancer cells can selfishly exploit nutrient supply, since nutrient abundance stimulates the production of growth factors, increasing cell growth and division (Han et al., 2015; Narita et al., 2019; Tucci, 2012). Conversely, nutrient restriction promotes the conservation of resources through autophagy, apoptosis, and decreased cell division, thereby protecting against cancer cell proliferation (Hsieh et al., 2005; Takakuwa et al., 2019; Tucci, 2012). These findings suggest that in some group contexts, resource availability can be altered to promote either cooperation or selfishness. However, since cooperative and selfish entities compete at different levels of selection, both within and between groups, it is crucial to integrate the study of selection across these different levels to more fully understand how vital resources shape the competition dynamics between cooperative and selfish entities.

We sought to investigate the relationship between resource availability and multilevel 
selection on selfish and cooperative mitochondria. Cells normally contain dozens to thousands of mitochondrial organelles that undergo dynamic fusion and fission (Tilokani et al., 2018). These organelles in turn contain multiple copies of mitochondrial DNA (mtDNA), which are usually non-recombining and can replicate throughout the cell cycle (Chatre and Ricchetti, 2013; Newlon and Fangman, 1975; Sena et al., 1975). Mitochondria cooperate with each other and with their host by supplying energy; in return, the nuclear genome supplies the proteins and building blocks needed to replicate mtDNA. Selfish mtDNA can be defined as mutant variants that successfully propagate at the expense of host fitness (Taylor et al., 2002). Such mutants can coexist with their cooperative counterparts for many generations in a heteroplasmic state, hence multilevel selection shapes the population dynamics of mtDNA (Dubie et al., 2020; Klucnika and Ma, 2019; Rollins et al., 2016; Shou, 2015; Taylor et al., 2002). In yeast cells, for example, respiratory-deficient mutant mtDNA can outcompete wildtype mtDNA, resulting in the formation of "petite" colonies that fail to grow on nonfermentable food sources (Gaillard and Bernardi, 1979; Goldring et al., 1971; MacAlpine et al., 2001). In Drosophila, dysfunctional mtDNA copies can outcompete their functional counterparts within individual flies to the point of driving the fly stock extinct (Ma and O'Farrell, 2016). Some evidence suggests that selfish mtDNA might even persist in a heteroplasmic state over evolutionary timescales. For example, the same selfish mtDNA mutation has been identified in multiple geographically isolated strains of the nematode species Caenorhabditis briggsae (Howe and Denver, 2008). 
How does resource availability influence the selection acting on selfish mtDNA? We address this question using a selfish mutant mtDNA within the model species Caenorhabditis elegans. First, we isolate and measure selection on the mutant mtDNA separately within individual hosts (sub-organismal) and between hosts (organismal). Consistent with the predictions of multilevel selection, sub-organismal selection favors the mutant mtDNA over the cooperative wildtype mtDNA, while the reverse is true at the level of organismal selection. Next, we applied this approach to study the effects of dietary nutrient availability and the physiology of metabolic stress tolerance. We show that although diet and nutrient sensing govern overall mtDNA levels, the preferential proliferation of $\triangle \mathrm{mtDNA}$ at the sub-organismal level depends on the Forkhead box $\mathrm{O}$ (FoxO) transcription factor DAF-16. At the level of organismal selection, diet restriction promotes the relative fitness of the cooperative over the selfish mtDNA, but only in the absence of FoxO. This observation indicates that FoxO promotes host tolerance to the selfish mtDNA during food scarcity. Broadly speaking, these findings suggest that resilience to food scarcity influences the relative fitness of cooperators and cheaters both within and between groups.

\section{Results}

\section{An experimental strategy to isolate selection on a selfish mitochondrial genome} at different levels

Selection can act directly on individual mtDNA molecules within an organelle due to intrinsic replication advantage (Holt et al., 2014). Selection can also occur between organelles within a host cell (Lieber et al., 2019; Zhang et al., 2019), between cells 
within a multicellular host (Shidara et al., 2005), and finally between host organisms. The vast majority of mitochondrial content in the adult hermaphroditic nematode Caenorhabditis elegans is confined to the germline (Bratic et al., 2009), which exists as a contiguous syncytium of cytoplasm until the final stages of oocyte maturation (Pazdernik and Schedl, 2013). Sub-organismal selection thus predominantly reflects the biology of the female germline, where mtDNA variants compete for transmission to the next generation. Accordingly, here we focus on selection at the sub-organismal level as a single phenomenon, in addition to selection at the organismal level.

The present study takes advantage of the well-characterized heteroplasmic mutant genome uaDf5 (Figure 1A), hereinafter referred to as $\triangle$ mtDNA (Ahier et al., 2018; Gitschlag et al., 2016; Liau et al., 2007; Lin et al., 2016; Tsang and Lemire, 2002). This deletion mutation spans four protein-coding genes and seven tRNA genes, disrupting gene expression and metabolic function. To quantify sub-organismal selection, we modified a previous approach to measure $\triangle$ mtDNA frequency in parents and their respective progeny as a function of parental $\triangle$ mtDNA frequency (Tsang and Lemire, 2002). Mutant frequency was measured longitudinally at successive developmental stages and across multiple parent-progeny lineages, which were propagated in isolation from one another to minimize the effect of organismal selection on overall $\triangle \mathrm{mtDNA}$ frequency (Figures $1 \mathrm{C}-1 \mathrm{D}$ and S1). Initially, we observe reduced $\triangle \mathrm{mtDNA}$ frequency in embryos compared to their parents (Figure 1B and 1C), consistent with germline purifying selection (Ahier et al., 2018; Hill et al., 2014; Lieber et al., 2019; Ma et al., 2014; Stewart et al., 2008). However, $\Delta$ mtDNA proliferates across development, 
achieving even higher frequency on average in adult progeny than in their respective parents (Figure 1C and 1D). Overall, we have quantitatively measured the frequencydependent selection acting on $\triangle \mathrm{mtDNA}$ at the sub-organismal level (Figure 1D).

We observe several indicators that $\triangle$ mtDNA compromises host fitness (Figure 2A-2C), in agreement with previous studies of this genome (Gitschlag et al., 2016; Liau et al., 2007; Lin et al., 2016). To quantify selection against $\Delta$ mtDNA strictly at the level of host fitness, we competed heteroplasmic animals carrying $\triangle \mathrm{mtDNA}$ against their homoplasmic wildtype counterparts on the same food plate (Figure 2D). In parallel, we propagated non-competing lines (lacking wildtype animals) to control for the confounding influence of sub-organismal heteroplasmy dynamics. By normalizing the population-wide changes in $\triangle$ mtDNA frequency to that of the non-competing control lines, we could measure selection against $\triangle$ mtDNA at the organismal level via the relative decline in $\triangle \mathrm{mtDNA}$ frequency across the competing lines (Figures $2 \mathrm{E}$ and S2).

We sought to integrate these measurements of organismal and sub-organismal selection into a single mathematical framework to describe the overall population dynamics of $\triangle$ mtDNA. The Price equation provides such a framework by describing evolution in terms of the covariance between a measurable trait value and its associated reproductive fitness (Frank, 1997; Price, 1972). Since the Price equation can accommodate more than one source of covariance, it is ideal for characterizing selection at multiple levels:

$$
w \Delta z=\operatorname{cov}(w, z)_{o r g}+E\left[\operatorname{cov}(w, z)_{s u b}\right]
$$


Here, $z$ refers to a character trait, in this case mitochondrial genotype. Since mitochondrial genotype consists of a heteroplasmic mix of $\triangle$ mtDNA and wildtype mtDNA, we define the trait value $z$ as the heteroplasmic frequency of $\triangle \mathrm{mtDNA}$, while $w$ refers to the fitness or reproductive rate of $\triangle$ mtDNA relative to that of wildtype mtDNA. The subscripts org and sub label the covariance between $w$ and $z$ at the organismal and sub-organismal levels, respectively. The expression $E$ refers to expected, or average, sub-organismal covariance between $w$ and $z$. Moreover, the strength with which natural selection acts on a genotype can be expressed as the selection coefficient, $s$ (equal to $1-w)$. Hence, the change in a trait due to selection can be described using the covariance between trait value and selection coefficient. Summing the organismal and sub-organismal covariances then provides a description of the overall population-level selection dynamics of $\triangle \mathrm{mtDNA}$.

We converted the measured sub-organismal shifts in $\triangle$ mtDNA frequency per generation (Figure 1D) to selection coefficients and plotted them as a function of parental $\Delta \mathrm{mtDNA}$ frequency (Figure 3, top graph). At a heteroplasmic $\triangle$ mtDNA frequency of approximately $60 \%$, we found that the organismal selection coefficient for selection against $\triangle$ mtDNA is $0.23 \pm 0.04$ (95\% C.I.) across 8 replicate lineages (Figures $2 \mathrm{E}$ and S2A). The organismal selection coefficient would be zero when $\triangle$ mtDNA frequency is $0 \%$, and the selection coefficient would be 1.0 when $\triangle$ mtDNA frequency is $100 \%$ since the mutation deletes essential genes and is thus predicted to be lethal at $100 \%$. Based on these data points, we derived a non-linear regression between $\triangle \mathrm{mtDNA}$ frequency and selection coefficient (Figure 3, middle graph). This regression predicts that the 
organismal fitness cost accelerates with increasing $\triangle$ mtDNA frequency, which is consistent with the notion of a phenotypic threshold effect as mutant mtDNA levels rise (Letellier et al., 1994; Picard et al., 2014; Sciacco et al., 1994). Summing the suborganismal and organismal regressions yields the overall population-level relationship between $\triangle$ mtDNA frequency and selection (plotted in Figure 3, bottom graph):

$$
s_{p}=[(0.0115 \pm 0.0061)(z)-(0.92 \pm 0.35)]_{s u b}+\left[10^{(0.0161 \pm 0.0022)(z)-1.61 \pm 0.20}\right]_{\text {org }}
$$

Here, $s_{p}$ represents the overall (population-level) selection coefficient against $\Delta$ mtDNA while the subscripts sub and org label the sub-organismal and organismal regressions, respectively.

To derive this model, we assumed that $\triangle$ mtDNA becomes non-viable upon reaching $100 \%$ frequency. However, $\triangle$ mtDNA is rarely observed above $90 \%$ frequency (Gitschlag et al., 2016; Liau et al., 2007). To account for the uncertainty in how organismal selection changes with rising $\triangle \mathrm{mtDNA}$ frequency, we set the organismal selection coefficient to 1.0 when $\triangle$ mtDNA frequency reaches $90 \%$ (Figure 3, gray shaded region). We also tested the assumption that organismal selection increases linearly with $\triangle$ mtDNA frequency (Figure S3). These alternate models had negligible effect on our overall conclusions. In each case, positive sub-organismal selection and negative organismal selection balance when $\triangle \mathrm{mtDNA}$ is near $60 \%$ mean heteroplasmic frequency. In summary, we have separately measured selection on $\triangle \mathrm{mtDNA}$ at each level and integrated them into the mathematical framework of the Price equation, which robustly predicts the population-level dynamics of $\triangle \mathrm{mtDNA}$ that we observe. 


\section{$\Delta$ mtDNA exploits nutrient status to propagate}

Using this framework, we sought to investigate how resource availability affects the multilevel selection acting on $\triangle \mathrm{mtDNA}$. Given the central role of mitochondria in metabolism, we reasoned that the multilevel selection acting on $\triangle$ mtDNA might be particularly sensitive to dietary perturbations. We therefore raised nematodes on food plates seeded with either a high or low concentration of E. coli (OP50 strain), which were UV-killed to prevent further bacterial growth (Figure S5A-S5B). UV-killed OP50 partially mimics diet restriction (Win et al., 2013). Nevertheless, we found that nematodes raised on the more restricted (low concentration) diet during the transition from larvae to mature adulthood-when the greatest net increase in mtDNA copy number occurs-harbored significantly lower $\triangle$ mtDNA frequency compared to those raised on a more abundant control (high concentration) diet (Figure 4A). Moreover, we observed that sub-organismal $\triangle$ mtDNA frequency is even higher when animals are raised on live food (Figure S5C). Tracking mutant frequency across development revealed that $\triangle \mathrm{mtDNA}$ levels rise from embryos to adults on a control diet as expected, but not in animals grown on a restricted diet (Figure $4 \mathrm{~B}$ and $4 \mathrm{C}$ ). Based on these observations, we conclude that $\triangle \mathrm{mtDNA}$ exploits nutrient abundance to rise in frequency during development but fails to do so under conditions of diet restriction.

\section{Insulin signaling influences sub-organismal proliferation of $\Delta$ mtDNA}

To better understand the role of nutrient availability in sub-organismal $\triangle m t D N A$ proliferation, we targeted the insulin-signaling pathway. Insulin acts as a nutrientdependent growth hormone and regulator of metabolic homeostasis, tailoring the 
appropriate physiological responses to external nutrient conditions (Badisco et al., 2013; Danielsen et al., 2013; Das and Arur, 2017; Lee and Dong, 2017; Lopez et al., 2013; Michaelson et al., 2010; Porte et al., 2005; Puig and Tjian, 2006; Shiojima et al., 2002). Nematodes expressing a defective allele of the insulin receptor homolog daf-2 perceive starvation, even in presence of food. However, disrupting insulin signaling in young larvae causes the dauer phenotype, a form of developmental arrest (Gottlieb and Ruvkun, 1994). Thus, we cannot directly track $\Delta$ mtDNA frequency across development under prolonged loss of daf-2 function. Instead, we used a temperature-sensitive daf-2 allele to bypass dauer arrest. We incubated these animals at a permissive temperature $\left(16^{\circ} \mathrm{C}\right)$ during early larval development, preserving insulin signaling. Next, we shifted these animals to the restrictive temperature $\left(25^{\circ} \mathrm{C}\right)$ at a later larval $(\mathrm{L} 4)$ stage, thereby disrupting insulin signaling during the transition from larva to mature adult. In control animals expressing a wildtype receptor, adult maturation at $25^{\circ} \mathrm{C}$ coincides with rising $\triangle m t D N A$ frequency compared to maturation at $16^{\circ} \mathrm{C}$ (Figure 4E). However, this dramatic increase in $\triangle$ mtDNA frequency is abolished in temperature-sensitive daf-2 mutants (Figure 4E). Indeed, we observed no overall change in average $\triangle$ mtDNA frequency across four independent lineages of the temperature-sensitive daf-2 mutants even after four consecutive generations of adult maturation at $25^{\circ} \mathrm{C}$ and subsequent recovery of larval progeny at $16^{\circ} \mathrm{C}$ (Figure S5D). In contrast, we observed a robust increase in $\triangle m t D N A$ frequency in wildtype controls. We therefore conclude that nutrient sensing via the insulin-signaling pathway regulates the sub-organismal proliferation of $\triangle \mathrm{mtDNA}$. 
The insulin receptor communicates nutrient status to the cell largely through the negative regulation of the FoxO family of transcription factors (O-Sullivan et al., 2015), encoded by the gene daf-16 in C. elegans (Figure 4D). Nutrient limitation or inactivation of the receptor activates FoxO/DAF-16, resulting in altered expression of its target genes. We therefore hypothesized that DAF-16 functions downstream of DAF-2 to suppress $\triangle$ mtDNA proliferation. Indeed, deletion of daf-16 restores the temperaturedependent proliferation of $\triangle \mathrm{mtDNA}$ in animals expressing the temperature-sensitive daf2 allele (Figure 4E). These data support a role for DAF-16 in regulating $\triangle \mathrm{mtDNA}$ proliferation.

Next, we sought to understand how DAF-2-dependent inhibition of DAF-16 regulates $\triangle m t D N A$ proliferation. In addition to $\triangle$ mtDNA frequency, we also found that total mtDNA copy number rises at $25^{\circ} \mathrm{C}$ compared to $16^{\circ} \mathrm{C}$ (Figure $4 \mathrm{~F}$ ). Interestingly, increased copy number of $\triangle \mathrm{mtDNA}$ entirely accounts for the rise in total copy number. Furthermore, higher total copy number at $25^{\circ} \mathrm{C}$ was abolished in daf-2 mutants and restored in daf2;daf-16 double-mutants (Figure 4F). To understand whether rising total mtDNA copy number promotes $\triangle$ mtDNA proliferation, or merely results from it, we quantified copy number across the same conditions in animals lacking $\triangle$ mtDNA. Homoplasmic-wildtype mtDNA copy number rose at $25^{\circ} \mathrm{C}$ compared to $16^{\circ} \mathrm{C}$ (Figure $4 \mathrm{G}$ ). This rise in copy number was suppressed in daf-2 mutants and partially but significantly rescued in daf2; daf-16 double-mutants (Figure 4G). Moreover, RNAi knockdown of daf-2 expression also suppressed mtDNA copy number in a daf-16-dependent manner (Figure S5E). Together, these data show that DAF-2 signaling inhibits DAF-16 to allow high mtDNA 
copy number, which permits sub-organismal $\triangle$ mtDNA proliferation at the warmer temperature.

How might DAF-16 suppress mtDNA copy number upon loss of DAF-2 signaling? DAF16 could achieve copy-number suppression via elimination of mitochondria, either through mitochondrial fragmentation and subsequent mitochondrial autophagy, or at the cellular level through apoptosis. However, these processes do not appear to play a role in mtDNA copy number suppression in daf-2 mutants (Figures 5A-5C and S6). Alternatively, DAF-16 might suppress mtDNA copy number by limiting mitochondrial biogenesis. Nutrient availability and insulin signaling each promote development of the germline (Angelo and Van Gilst, 2009; Drummond-Barbosa and Spradling, 2001; Michaelson et al., 2010; Narbonne and Roy, 2006; Shim et al., 2002), which harbors the vast majority of mtDNA in the adult nematode (Bratic et al., 2009). We observed that mitochondrial organelle quantity and mtDNA copy number are proportional to gonad size across wildtype, daf-2 mutant, and daf-2;daf-16 double-mutants (Figure 5D-5G). These data indicate that upon loss of insulin signaling, DAF-16 suppresses germline development and the accompanying biogenesis of mtDNA.

The observation that DAF-16 suppresses mtDNA copy number in insulin-signaling mutants suggests that DAF-16 may also suppress mtDNA copy number in response to diet restriction. However, total mtDNA copy number was substantially lower in heteroplasmic animals raised on the restricted diet compared to the control diet, even in daf-16 mutants (Figure 6A). These data show that diet restriction limits copy number 
independently of DAF-16. Given that elevated copy number is associated with higher $\triangle m t D N A$ frequency (Figure $4 \mathrm{E}$ and $4 \mathrm{~F}$ ), $\triangle \mathrm{mtDNA}$ frequency may also benefit from dietary nutrient abundance independently of DAF-16. Remarkably, $\Delta$ mtDNA frequency was higher on the more plentiful control diet compared to the restricted diet, but only when DAF-16 was present (Figure 6B). Moreover, while total mtDNA copy number and $\triangle m t D N A$ frequency each rose significantly across development on the control diet compared to the restricted diet (Figure 6C), copy number increased without any accompanying change in $\triangle$ mtDNA frequency in daf-16 mutants (Figure 6D). Together, these data reveal two opposing roles for DAF-16. Upon loss of insulin signaling, DAF-16 suppresses germline development and mtDNA copy number, limiting the niche space in which $\triangle$ mtDNA can proliferate. Conversely, DAF-16 is required for preferential $\triangle m t D N A$ proliferation under conditions of nutrient abundance. We therefore conclude that nutrient abundance and DAF-16 are each necessary, but not sufficient individually, for the suborganismal selection advantage of $\triangle \mathrm{mtDNA}$.

\section{Nutrient status governs selection on $\triangle$ mtDNA at different levels}

Dietary nutrient abundance and DAF-16 each affect sub-organismal $\triangle$ mtDNA frequency (Figure 6A-6D), and $\triangle$ mtDNA frequency itself affects host fitness. Additionally, FoxO/DAF-16 regulates numerous genes involved in stress tolerance (Klotz et al., 2015; Martins et al., 2016; Murphy et al., 2003; Tepper et al., 2013; Webb et al., 2016) and promotes organismal survival during nutrient scarcity (Greer et al., 2007; Hibshman et al., 2017; Kramer et al., 2008). Given these considerations, we hypothesized that dietary nutrient availability and DAF-16 affect selection on $\triangle \mathrm{mtDNA}$ at both the 
organismal and sub-organismal levels. Sub-organismal selection was quantified as before (see Figure 1D), under restricted versus control diets, in the presence versus absence of DAF-16 (Figure 7A and 7B). Organismal selection was quantified under each of these same conditions, using the competition method previously described (see Figure 2D and 2E). In populations with wildtype DAF-16, diet restriction did not significantly affect the decline in $\triangle$ mtDNA frequency at the level of organismal selection (Figures 7C, S7A and S7D). However, diet restriction accelerated the selection against $\triangle m t D N A$ at the organismal level in competing populations of daf-16 mutants (Figures 7D, S7B, S7C and S7E). These data indicate that on a restricted diet, DAF-16 helps curtail organismal selection against $\triangle \mathrm{mtDNA}$.

Finally, we used the framework of the Price equation to integrate the sub-organismal and organismal covariances for each of the four conditions tested (Figure S7D and S7E). The combined covariances predict that the strongest overall selection against $\triangle m t D N A$ occurs in populations lacking DAF-16 experiencing food scarcity, and the weakest overall selection occurs in populations with DAF-16 experiencing food abundance, with the remaining two conditions each experiencing an intermediate strength of selection (Figure S7D and S7E, bottom graphs). Measuring $\triangle$ mtDNA frequency across non-competing heteroplasmic populations afforded the opportunity to test this prediction. Remarkably, this prediction is consistent with our observation (Figure 7E). Combined, our data show that quantitatively accounting for selection at each level allows for a fuller description of the population dynamics of a cheater 
genome, including the effects of food scarcity and host genotype on those dynamics (Figure 7F).

\section{Discussion}

Deleterious fitness effects are no guarantee that natural selection will remove a trait from the population. On the contrary, traits that are deleterious at one level of scale can be favored at a different level, a phenomenon known to characterize mtDNA heteroplasmy dynamics (Clark et al., 2012; Dubie et al., 2020; Gitschlag et al., 2016; Liau et al., 2007; Lin et al., 2016; Ma and O'Farrell, 2016; Taylor et al., 2002; Tsang and Lemire, 2002). By isolating and measuring selection at each level, we have applied a theoretical framework to empirical data to uncover the impact of environmental context on multilevel selection. Using this approach, we identified a diet-by-genotype interaction that influences the population genetics of mitochondria in C. elegans.

We have shown here that although dietary nutrient abundance fuels mtDNA replication, the preferential sub-organismal proliferation of $\triangle$ mtDNA depends on DAF-16 and its downstream functions. Our study provides generalizable insights into conditions that determine the fitness of cheaters versus cooperators. In particular, we find that the opportunity for cheaters to outcompete cooperators requires resources and a niche space where replication can occur. However, while necessary, these conditions are not sufficient to ensure the fitness advantage of cheaters. On the contrary, additional conditions shape the cost of cooperation, or the benefit of cheating, and hence the fitness advantage of cheaters. 
How does DAF-16 promote $\triangle$ mtDNA proliferation? This could occur via several potential mechanisms. For example, a recent study in heteroplasmic flies found that PINK1 preferentially localizes to mitochondria enriched for the mutant genome in ovaries, inhibiting protein synthesis and mtDNA replication (Zhang et al., 2019). Furthermore, previous genome-wide expression analysis identified DAF-16 as a potential negative regulator of the gene encoding PINK1 (Tepper et al., 2013), suggesting that DAF-16 might interfere with the PINK1-dependent preferential replication of wildtype mtDNA, although it is not known whether this function of PINK1 is evolutionarily conserved between flies and nematodes. Another recent study from the same group found that insulin receptor signaling functions through the protein Myc to limit the propagation of mutant mtDNA in heteroplasmic fly ovaries (Wang et al., 2019). Interestingly, Myc has also been identified among the target genes of FoxO transcription factors in the fly soma (Teleman et al., 2008) as well as in mammals (Webb et al., 2016), suggesting that DAF-16 might function through a similar mechanism to regulate heteroplasmy dynamics in nematodes. Alternatively, DAF-16 could promote $\triangle$ mtDNA proliferation via the alleviation of bioenergetic stress. DAF-16 up-regulates the expression of multiple genes involved in energy metabolism, including several glycolytic enzymes (Depuydt et al., 2014; Tepper et al., 2013). By promoting the mitochondria-independent production of ATP, DAF-16 might be contributing to metabolic conditions that make the cell more tolerant to the presence of mutant mitochondria. This possibility is consistent with previous work implicating other compensatory stress-response mechanisms in the proliferation of $\triangle$ mtDNA (Gitschlag et al., 2016; Lin et al., 2016). 
How does resource availability influence selection on cooperators versus cheaters at the level of competing groups? Note that the female germline, along with the cell lineage that develops from an oocyte to a germline in the next generation, harbors the mtDNA molecules that compete for transmission. Organismal selection acting on mtDNA can thus be viewed as a group-level phenomenon, while sub-organismal selection represents the within-group level. On one hand, if resource scarcity selects for cooperation, then groups with a higher proportion of cooperators should experience an additional fitness advantage over other groups during times of scarcity. On the other hand, exposure to cheating can result in an evolutionary arms race in which cooperators acquire resistance to cheaters, a phenomenon observed in bacteria and social amoebae (Hollis, 2012; Khare et al., 2009; O'Brien et al., 2017). Could food scarcity select for adaptations that reduce the fitness impact of metabolic cheaters? We propose that DAF-16 represents an example of this type of stress tolerance. Although diet restriction compromised the sub-organismal advantage of $\triangle \mathrm{mtDNA}$, it had no effect on the organismal disadvantage, provided DAF-16 is present. However, in daf-16 mutants, diet restriction intensified organismal selection against $\triangle$ mtDNA. FoxO/DAF-16 is known to promote survival under conditions of nutrient limitation (Greer et al., 2007; Hibshman et al., 2017; Kramer et al., 2008). Here, we have shown that the same gene also promotes host tolerance to the presence of $\triangle$ mtDNA during food scarcity.

It is important to note that we do not draw specific inferences about the genetic composition of naturally occurring populations. For example, this study does not 
assume that nuclear or mitochondrial mutants would necessarily self-segregate into separate populations in the wild. Likewise, organisms carrying a deleterious heteroplasmy are unlikely to spread through a population except in rare circumstances, such as following an extreme population bottleneck. Rather, we sought to uncover basic principles describing how resource availability and multilevel selection interact to shape the population dynamics of cooperative and selfish entities. In conclusion, the work presented here suggests that when cooperators and cheaters compete across different levels of selection, resource availability-and resilience to resource scarcity-together shape the proliferative capacity of the cheaters, as well as the ability of the cooperators to collectively tolerate their presence.

\section{Acknowledgments}

We thank the members of the Patel Laboratory (James P Held, Cait S Kirby, Nikita Tsyba, Benjamin Saunders, Cassidy A Johnson), Janet M Young, Mia T Levine, Sarah E Zanders, Harmit S Malik, and Antonis Rokas for their valuable feedback on the manuscript. This work was generously supported by R01 GM123260 (M.R.P.), the Ruth

L. Kirschstein National Research Service Award Individual Predoctoral Fellowship 1F31GM125344 (B.L.G.), and the Vanderbilt University Medical Center Diabetes Research and Training Center Pilot and Feasibility Grant. Confocal microscopy imaging was performed through the use of the Vanderbilt Cell Imaging Shared Resource (supported by NIH grants CA68485, DK20593, DK58404, DK59637 and EY08126). Quantification of mtDNA copy number and $\triangle \mathrm{mtDNA}$ frequency was conducted with the help of the Simon A. Mallal Laboratory at Vanderbilt University Medical Center. 


\section{Author contributions}

Conceptualization, B.L.G. and M.R.P; Methodology, B.L.G, A.T.T., and M.R.P.; Formal Analysis, B.L.G, A.T.T., and M.R.P.; Investigation, B.L.G.; Resources, M.R.P.; Writing Original Draft, B.L.G.; Writing - Review \& Editing, B.L.G, A.T.T., and M.R.P.; Visualization, B.L.G.; Supervision, M.R.P.; Funding Acquisition, B.L.G. and M.R.P.

\section{Figure 1. Quantification of sub-organismal selection for $\triangle$ mtDNA}

(A) Map of $C$. elegans mtDNA showing the deletion that specifies the $\triangle \mathrm{mtDNA}$ variant, uaDf5 (dark red). Genes are color-coded according to functional category: respiratory complex I (light red), complex III (yellow), complex IV (light blue), complex V (dark blue), ribosomal RNA (gray), tRNA (black), non-coding regions (thin line).

(B) Frequency distributions of $\triangle \mathrm{mtDNA}$ across three life stages of single broods from parents with low (top, $\mathrm{N}=94$ ), intermediate (middle, $\mathrm{N}=93$ ), or high (bottom, $\mathrm{N}=88$ ) $\Delta \mathrm{mtDNA}$ frequency. Dotted lines represent parent $\triangle \mathrm{mtDNA}$ frequency.

(C) $\triangle$ mtDNA frequency between individual parents and age-synchronized progeny at three life stages. Each line is an individual parent-progeny lineage. Because parental $\triangle$ mtDNA frequency impacts fecundity and development (Figure 2C and 2D), individual parent-progeny lineages were propagated in isolation in order to minimize the effects of organismal fitness and magnify the effect of sub-organismal selection. Each parental data point represents a single parent lysed individually; each progeny data point represents three age-synchronized progeny lysed together to also minimize the effect of random drift. Friedman test with Dunn's multiple comparisons test. 
(D) Shift in $\triangle$ mtDNA frequency per generation, between parents and their respective age-matched adult progeny. Plotted as a function of parental frequency using first and last time-points in (C). Red shaded region represents 95\% C.I.

\section{Figure 2. Quantification of organismal selection against $\triangle$ mtDNA}

(A) Basal aerobic respiration and maximum respiratory capacity in age-synchronized adults maintaining $\triangle$ mtDNA. Two-way ANOVA with Sidak's multiple comparisons test.

(B) Peak fecundity as measured by viable progeny produced per hour from agesynchronized adults. Mitochondrial genotype was binned according to animals sampled from either the low end of the $\triangle$ mtDNA frequency distribution (defined as below the population mean of $60 \%, \mathrm{~N}=12$ ), or the high end (defined as above the population mean of $60 \%, N=35)$, with wildtype controls $(\mathrm{N}=8)$. Brown-Forsythe and Welch ANOVA with Dunnett's T3 multiple comparisons test.

(C) $\triangle$ mtDNA frequency between larval stages among a chronologically synchronized cohort. $\mathrm{N}=35$ per developmental stage. Brown-Forsythe and Welch ANOVA with Dunnett's T3 multiple comparisons test. Each data point represents one nematode.

(D) Schematic illustrating experimental design for competition experiment to quantify organismal selection against $\triangle$ mtDNA. Heteroplasmic carriers of $\triangle \mathrm{mtDNA}$ used in competing and non-competing lineages were taken from the same stock.

(E) Population-wide $\triangle$ mtDNA frequency, relative to $\triangle \mathrm{mtDNA}$ frequency per heteroplasmic individual, measured for 10 generations across 8 replicate competed (red) and non-competed (gray) lineages. Competed lineages consisted of $\Delta$ mtDNAcarrying heteroplasmic individuals mixed with homoplasmic wildtype counterparts on the 
same food plates. Non-competed lineages consisted of exclusively $\triangle \mathrm{mtDNA}$-carrying heteroplasmic individuals. Lower initial population-wide $\Delta$ mtDNA frequency in competing lines is due to the presence of wildtype animals. At each generation, population-wide $\triangle$ mtDNA frequency of competing and non-competing lines were sampled and normalized to average population-wide frequency of all non-competing lines (which is equivalent to average frequency per heteroplasmic individual, since all individuals within non-competing lines carry $\triangle \mathrm{mtDNA})$. Solid lines represent best-fit regressions across all replicate lineages.

Error bars represent 95\% C.I.

Figure 3. Fitness of wildtype mtDNA relative to $\triangle \mathrm{mtDNA}$ as a function of $\triangle \mathrm{mtDNA}$ frequency, at each selection level

Selection coefficient against $\triangle$ mtDNA at sub-organismal (top), organismal (middle), and overall combined (bottom) levels of selection. The top graph represents the same regression as in Figure $1 \mathrm{D}$ but with absolute $\triangle \mathrm{mtDNA}$ frequency shift converted to selection coefficient. When selection coefficient against $\triangle$ mtDNA is negative, positive selection is acting on $\triangle \mathrm{mtDNA}$, hence frequency rises. At $100 \% \Delta \mathrm{mtDNA}$ frequency, the mutation is lethal and hence the selection coefficient against $\triangle \mathrm{mtDNA}$ is expected to be 1.0 (middle graph, upper right corner). At $0 \%$ frequency, no $\Delta \mathrm{mtDNA}$ is available for selection, hence the organismal selection coefficient against $\triangle$ mtDNA is 0 . At $60 \%$ $\triangle m t D N A$ frequency, the empirically derived selection coefficient against $\triangle \mathrm{mtDNA}$ is 0.23 (95\% C.I. \pm 0.04$)$. The plotted curve represents the line that passes through all three of these values for selection coefficient. The blue shaded region represents the range of 
$\triangle m t D N A$ frequency in which the organismal and sub-organismal selection coefficients balance, resulting in a stably persisting $\triangle$ mtDNA near $60 \%$ frequency. Red regions represent 95\% C.I. Gray shaded regions (middle and lower graphs) show regressions when selection coefficient reaches 1.0 at $90 \% \Delta \mathrm{mtDNA}$ frequency.

Figure 4. $\triangle$ mtDNA exploits nutrient supply and insulin signaling to proliferate at the sub-organismal level.

(A) Frequency of $\triangle$ mtDNA between age-synchronized adults raised on a restricted versus control diet. Mann-Whitney test. $\mathrm{N}=8$ lysates containing 5 pooled agesynchronized adults per lysate.

(B-C) $\triangle$ mtDNA levels in individual parents and age-synchronized progeny at two life stages, embryo and mature adult, across lineages raised on either control (B) or restricted $(\mathrm{C})$ diet. Each parental data point represents a single parent lysed individually; each progeny data point represents three age-synchronized progeny lysed together. Friedman test with Dunn's multiple comparisons test. $\mathrm{N}=24$ parent-progeny lineages (control diet); N=20 parent-progeny lineages (restricted diet).

(D) Schematic of the FoxO-dependent insulin signaling cascade, with C. elegans homologs of mammalian proteins in parentheses.

(E) Frequency of $\triangle$ mtDNA in age-synchronized adults of wildtype, temperature-sensitive daf-2(e1370) mutant, null daf-16(mu86) mutant, or double-mutant genotype. Nematodes were raised from late larval $(\mathrm{L} 4)$ to mature adulthood at $25^{\circ} \mathrm{C}$, the restrictive temperature for daf-2(e1370), with controls raised at the permissive temperature of $16^{\circ} \mathrm{C}$. Two-way 
ANOVA with Bonferroni correction. $\mathrm{N}=8$ lysates containing 5 pooled age-synchronized adults each.

(F) mtDNA copy number of the same samples shown in (E), separated by mitochondrial genotype and normalized to total mtDNA of adults with the wildtype nuclear genotype raised at the control temperature of $16^{\circ} \mathrm{C}$. Wildtype mtDNA copy number is shown in green, total mtDNA copy number is shown in purple. The vertical distance between wildtype and total mtDNA represents $\triangle$ mtDNA copy number. Two-way ANOVA with Bonferroni correction.

(G) mtDNA copy number in homoplasmic age-synchronized adults of wildtype, temperature-sensitive daf-2(e1370) mutant, null daf-16(mu86) mutant, or double-mutant genotype, raised from $L 4$ stage at the restrictive temperature of $25^{\circ} \mathrm{C}$, with controls maintained at the permissive temperature of $16^{\circ} \mathrm{C}$. Two-way ANOVA with Bonferroni correction. $\mathrm{N}=8$ lysates containing 5 pooled age-synchronized adults each.

Error bars represent 95\% C.I.

Figure 5. DAF-16 activation upon loss of insulin signaling suppresses mtDNA content via regulation of germline proliferation

(A-C) mtDNA copy number in age-synchronized adults of wildtype, temperaturesensitive daf-2(e1370) mutant, null daf-16(mu86) mutant, or double-mutant genotype, each raised from L4 stage at $25^{\circ} \mathrm{C}$. Copy number is also shown in wildtype, daf2(e1370), and daf-2(e1370);daf-16(mu86) double-mutant adults each with $p d r-1$ (gk448) (A), ced-3(ok2734) (B), or drp-1(tm1108) (C), representing loss-of-function alleles of the Parkin homologue, a terminator caspase, and dynamin-related protein, respectively. 
mtDNA copy number in daf-16(mu86) single-mutants is also shown. One-way ANOVA with Bonferroni correction. $\mathrm{N}=8$ lysates containing 5 pooled age-synchronized adults each.

(D-E) Images and quantification of germline mitochondrial organelle signal intensity as indicated by the fluorescent reporter TOMM-20::mCherry, across age-synchronized adults of wildtype, temperature-sensitive daf-2(e1370) mutant, null daf-16(mu86) mutant, or double-mutant genotype, raised from $\mathrm{L} 4$ stage at $25^{\circ} \mathrm{C}$. Each data point in (E) represents one age-synchronized adult nematode, shown in (D). One-way ANOVA with Bonferroni correction.

(F-G) Images and quantification of DAPI-stained nuclei and mtDNA copy number of age-synchronized adults of wildtype, temperature-sensitive daf-2(e1370) mutant, null daf-16(mu86) mutant, or double-mutant genotype, raised from L4 stage at $25^{\circ} \mathrm{C}$. Each nuclei data point in (G) represents one female gonad (age-synchronized adults); each mtDNA copy number data point represents a lysate containing 5 pooled agesynchronized adults. Two-way ANOVA with Bonferroni correction.

Error bars represent 95\% C.I.

Figure 6. The sub-organismal selection advantage of $\triangle$ mtDNA requires both nutrient abundance and DAF-16.

(A) Total mtDNA copy number across heteroplasmic age-synchronized adults of wildtype nuclear genotype or null daf-16(mu86) genotype, raised on a restricted diet versus a control diet. Two-way ANOVA with Bonferroni correction. $\mathrm{N}=8$ lysates containing 5 pooled age-synchronized adults each. 
(B) $\triangle$ mtDNA frequency across the samples shown in (A). Two-way ANOVA with Bonferroni correction.

(C-D) Shift in $\triangle$ mtDNA frequency across development as a function of change in mtDNA copy number, in control and restricted diets, across animals of wildtype nuclear genotype (C) or null daf-16(mu86) genotype (D). Each data point corresponds to an embryonic lysate (3 pooled embryos) and a corresponding adult lysate (3 pooled adults) taken from the same parent and lysed 96 hours apart. Mann-Whitney tests with Bonferroni correction. $\mathrm{N}=22$ (restricted diet, wildtype); $\mathrm{N}=24$ (control diet, wildtype); $\mathrm{N}=24$ (restricted diet, $\operatorname{daf}-16^{-/-}$); $\mathrm{N}=24$ (control diet, daf-16 ${ }^{-/-}$).

Error bars represent 95\% C.I.

\section{Figure 7. Nutrient status impacts multilevel selection dynamics of $\triangle \mathrm{mtDNA}$}

(A-B) Shift in $\triangle$ mtDNA frequency per generation attributable to sub-organismal mitochondrial dynamics, similar to Figure 1D, featuring parent-progeny lineages raised on a restricted or control diet, across animals of wildtype nuclear genotype (A) or null daf-16(mu86) genotype (B). Linear regression compares parental $\triangle$ mtDNA frequency with the magnitude of frequency-shift from parent to age-matched progeny. Regressions compared using analysis of covariance.

(C-D) Measure of organismal selection against $\triangle \mathrm{mtDNA}$, similar to Figure $2 \mathrm{~B}$, in competing lineages raised on a restricted or control diet, across animals of wildtype nuclear genotype (C) or null daf-16(mu86) genotype (D). Vertical axis represents relative $\triangle$ mtDNA frequency in the population, measured as proportion of average 
$\triangle m t D N A$ frequency per heteroplasmic individual (equal to population-wide $\Delta$ mtDNA frequency of non-competing populations).

(E) $\triangle$ mtDNA frequency, relative to starting frequency, in non-competing lineages from the organismal competition experiment shown in (C) and (D). Linear regression analyses with Bonferroni correction.

(F) Model illustrating the influence of DAF-16 on $\triangle$ mtDNA selection dynamics. Dietary nutrient supply and the inhibition of DAF-16 by insulin signaling promote germline growth and mtDNA replication (Figures 4 and 5). However, the ability of $\triangle$ mtDNA to take advantage of nutrient supply and preferentially proliferate requires DAF-16 (Figure 6), indicating that nutrient supply and DAF-16 are each necessary, but not sufficient individually, for $\triangle$ mtDNA proliferation. Organismal selection against $\triangle m t D N A$ intensifies during dietary restriction but only in the absence of DAF-16, indicating that DAF-16 partially shields $\triangle$ mtDNA from organismal selection during conditions of food scarcity. Hence, nutrient status and host genotype interact to shape the fitness of $\triangle \mathrm{mtDNA}$ across the levels of selection.

Figure S1, related to Figure 1. Quantification of mtDNA copy number and $\triangle \mathrm{mtDNA}$ frequency by droplet digital PCR

(A) Map of mtDNA showing the uaDf5 deletion ( $\triangle$ mtDNA) and the strategy for oligonucleotide primer design for the multiplex quantification of $\triangle \mathrm{mtDNA}$ and wildtype mtDNA simultaneously within the same reaction. Due to the deletion size, primers flanking the uaDf5 deletion (green arrows) amplify a PCR product off of the $\triangle \mathrm{mtDNA}$ but not wildtype mtDNA template (see panel B). Likewise, primers complementary to a 
sequence within the region spanning the uaDf5 deletion (blue arrows) amplify a PCR product off of the wildtype mtDNA but not $\triangle \mathrm{mtDNA}$ template.

(B-C) Sample droplet digital PCR data plots showing mtDNA copy number in lysates from homoplasmic wildtype (B) and heteroplasmic (C) nematodes. Mutant frequency is determined from $\triangle$ mtDNA copy number over total copy number.

Figure S2, related to Figure 2. Change in $\triangle \mathrm{mtDNA}$ frequency in competition experiments is due to organismal selection

(A) Quantification of $\triangle m t D N A$ frequency across 8 replicate competed (red) and noncompeted (black) lineages. Same experiment as shown in Figure 2A but with y-axis expressing raw $\triangle$ mtDNA frequency measurements. Dark lines reflect best-fit regression across all replicate lineages.

(B) Quantification of fraction of heteroplasmic members from the competition experiment shown in (A), based on gel images shown (C).

(C) PCR gel images reflecting individual adult nematodes sampled from the competition lines in the organism-level selection experiment. Approximately 48 adults were individually lysed from the first generation and every second generation thereafter. A single PCR band reflects Bristol strain nematodes (homoplasmic for wildtype mtDNA), whereas double bands reflect heteroplasmic animals carrying the $\triangle \mathrm{mtDNA}$ allele. The numbers to the sides of the gel images label the replicate lineages. 
Figure S3, related to Figure 3 . Fitness of wildtype mtDNA relative to $\triangle \mathrm{mtDNA}$ as a function of $\triangle$ mtDNA frequency, at each selection level, assuming organismal selection increases linearly with $\triangle$ mtDNA frequency

Selection coefficient against $\triangle$ mtDNA at sub-organismal (top), organismal (middle), and overall combined (bottom) levels of selection, similar to Figure 3 but assuming that the strength of organismal selection increases linearly with $\triangle \mathrm{mtDNA}$ frequency. The linear regression (gray) and the non-linear regression (red, also shown in Figure 3) of frequency-dependent organismal selection against $\triangle \mathrm{mtDNA}$ predict that sub-organismal and organismal selection cancel out near $60 \%$, allowing $\triangle$ mtDNA to persist in this range.

Figure S4, related to Figure 3. Description of the Price Equation and trait-fitness covariance

The Price Equation (top) shows that the change in a trait value $(\Delta z)$ is proportional to the covariance between the trait value itself and its fitness. Covariance describes the relationship between the variation in one value (e.g. trait) and variation in a separate value (e.g. fitness), assuming the two sets of values are correlated (bottom graph).

Figure S5, related to Figure 4. Diet and insulin signaling regulate mtDNA copy number and $\triangle$ mtDNA frequency

(A) Schematic of experimental workflow for assaying the impact of dietary and pharmacological perturbations on $\triangle \mathrm{mtDNA}$ frequency.

(B) Experimental conditions under which changes in $\triangle$ mtDNA frequency were assayed. 
(C) The frequency of $\triangle$ mtDNA in age-synchronized, pooled adults (10 adults per sample) grown on one of three food plates: UV-killed OP50 E. coli, live OP50 suspended in M9 buffer, and live OP50 suspended in LB medium. Kruskal-Wallis ANOVA with Dunn's multiple comparisons test.

(D) $\triangle$ mtDNA frequency in generation 1 versus generation 4 , between wildtype and the temperature-sensitive allele daf-2(e1370). Two-way ANOVA with Bonferroni correction. $\mathrm{N}=4$ lysates containing 5 pooled age-synchronized adults each.

(E) mtDNA copy number in age-synchronized adults lacking $\triangle$ mtDNA and expressing either wildtype or null daf-16(mu86), raised on either a daf-2 RNAi knockdown diet or an empty-vector control diet. Two-way ANOVA with Bonferroni correction. $\mathrm{N}=8$ lysates containing 5 pooled age-synchronized.

Error bars represent 95\% C.I.

Figure S6, related to Figure 5. No observed change in germline apoptosis between wildtype and daf-2 mutants

Images (A) and quantification (B) of apoptosis as indicated by the engulfment of apoptotic cells by the reporter CED-1::GFP (white arrows in A), between agesynchronized adults of wildtype or temperature-sensitive daf-2(e1370) mutant genotype. Mann-Whitney test.

Error bars represent 95\% C.I.

Figure S7, related to Figure 7. Quantification of multilevel selection between dietary conditions and daf-16 genotypes 
(A-B) Same data presented in Figure 7C-7E but with y-axis representing raw $\triangle$ mtDNA frequency measurements. Dark lines reflect best-fit regression across all replicate lineages.

(C) Fraction of $\triangle m t D N A$-carrying individuals at generation 8 of the competition experiment shown in (A) and (B), normalized to control-diet lines.

(D-E) Selection coefficients against $\triangle \mathrm{mtDNA}$ at sub-organismal (top graphs), organismal (middle graphs), and overall combined (bottom graphs) levels of selection, in restricted versus control diets, in animals with either a wildtype (D) or null daf16(mu86) (E) nuclear genotype. Shaded regions represent 95\% C.I. Dotted lines (middle and lower graphs) show regressions if selection coefficient is 1.0 at $90 \%$ $\Delta$ mtDNA frequency (confidence intervals omitted from these regressions for simplicity). Open-circle data points on overall regressions (bottom graphs) correspond to $\triangle \mathrm{mtDNA}$ frequency of non-competing lineages averaged across all 8 generations.

\section{STAR $\star M E T H O D S$}

\section{KEY RESOURCES TABLE}

\begin{tabular}{|l|l|l|}
\hline REAGENT or RESOURCE & SOURCE & IDENTIFIER \\
\hline \multicolumn{2}{|l|}{ Chemicals, Peptides, and Recombinant Proteins } & \\
\hline Blpl & New England Biolabs & Cat\#R0585L \\
\hline 2-deoxy-D-glucose & Sigma-Aldrich & Cat\#D8375-1G \\
\hline Isopropyl- $\beta$-D-thiogalactopyranoside & Research Products International & Cat\#I56000-1 \\
\hline 4',6-diamidino-2-phenylindole (DAPI) & Thermo Fisher Scientific & Cat\#D1306 \\
\hline Paraformaldehyde & Electron Microscopy Sciences & Cat\#15710 \\
\hline Levamisole & Fisher Scientific & Cat\# \\
& & AC187870100 \\
\hline Critical Commercial Assays & \multicolumn{2}{l|}{} \\
\hline DreamTaq Green DNA Polymerase & Thermo Fisher Scientific & Cat\#EP0713 \\
\hline
\end{tabular}




\begin{tabular}{|c|c|c|}
\hline $\begin{array}{l}\text { Eppendorf }{ }^{\mathrm{IM}} \text { 96-well twin.tec }{ }^{\mathrm{IM}} \text { PCR } \\
\text { plates }\end{array}$ & Fisher Scientific & Cat\#951020303 \\
\hline QX200 ddPCR EvaGreen Supermix & Bio-Rad & Cat\#1864034 \\
\hline $\begin{array}{l}\text { Automated Droplet Generation Oil for } \\
\text { EvaGreen }\end{array}$ & Bio-Rad & Cat\#1864112 \\
\hline $\begin{array}{l}\text { DG32 Automated Droplet Generator } \\
\text { Cartridges }\end{array}$ & Bio-Rad & Cat\#1864108 \\
\hline Droplet Reader Oil for ddPCR & Bio-Rad & Cat\#1863004 \\
\hline Seahorse XFe96 FluxPak & Agilent & Cat\#102601-100 \\
\hline \multicolumn{3}{|c|}{ Experimental Models: Organisms/Strains } \\
\hline $\begin{array}{l}\text { C. elegans wild isolate (C. elegans var } \\
\text { Bristol) }\end{array}$ & Caenorhabditis Genetics Center & N2 \\
\hline him-8(e1489); $\Delta \mathrm{mtDNA}(u a D f 5) /+$ & Bernard Lemire & LB138 \\
\hline daf-2(e1370) III & Jonathan A. Hodgkin & CB1370 \\
\hline daf-16(mu86) I & Cynthia J. Kenyon & CF1038 \\
\hline $\begin{array}{l}\text { daf-2(e1370) III; daf-16(mu86) I; } \\
\text { muEx268 [ges-1p::GFP::daf-16(cDNA })+ \\
\text { odr-1::RFP] }\end{array}$ & Caenorhabditis Genetics Center & CF1827 \\
\hline$p d r-1(\mathrm{gk} 448) \mathrm{III}$ & $\begin{array}{l}\text { International C. elegans Gene } \\
\text { Knockout Consortium }\end{array}$ & VC1024 \\
\hline$d r p-1(\operatorname{tm} 1108) \operatorname{IV}$ & Shohei Mitani & CU6372 \\
\hline ced-3(ok2734) IV & $\begin{array}{l}\text { International C. elegans Gene } \\
\text { Knockout Consortium }\end{array}$ & RB2071 \\
\hline bcls39 V [lim-7p::ced-1::GFP + lin-15(+)] & Barbara Conradt & MD701 \\
\hline tomm-20::mCherry II & Sasha de Henau & TBDL58 \\
\hline $\begin{array}{l}\text { unc-119(ed3); dkls398 [pie-1p::GFP::/gg- } \\
1+\text { unc-119(+)] }\end{array}$ & Ken Sato & GK682 \\
\hline $\begin{array}{l}\Delta \text { mtDNA }(\text { uaDf5)/+ crossed into Bristol } \\
\text { nuclear background }\end{array}$ & This study & $\mathrm{N} / \mathrm{A}$ \\
\hline daf-2(e1370) III; $\Delta \mathrm{mtDNA}(u a D f 5) /+$ & This study & N/A \\
\hline $\begin{array}{l}\text { daf-2(e1370) III; daf-16(mu86) I; } \\
\Delta \mathrm{mtDNA}(u a D f 5) /+\end{array}$ & This study & $\mathrm{N} / \mathrm{A}$ \\
\hline daf-16(mu86); $\Delta$ mtDNA(uaDf5)/+ & This study & N/A \\
\hline daf-2(e1370) III; pdr-1(gk448) III & This study & $\mathrm{N} / \mathrm{A}$ \\
\hline $\begin{array}{l}\text { daf-2(e1370) III; daf-16(mu86) I; pdr- } \\
\text { 1(gk448) III }\end{array}$ & This study & $\mathrm{N} / \mathrm{A}$ \\
\hline daf-2(e1370) III; drp-1(tm1108) IV & This study & $\mathrm{N} / \mathrm{A}$ \\
\hline $\begin{array}{l}\text { daf-2(e1370) III; daf-16(mu86) I; drp- } \\
\text { 1(tm1108) IV }\end{array}$ & This study & $\mathrm{N} / \mathrm{A}$ \\
\hline daf-2(e1370) III; ced-3(ok2734) IV & This study & $\mathrm{N} / \mathrm{A}$ \\
\hline $\begin{array}{l}\text { daf-2(e1370) III; daf-16(mu86) I; ced- } \\
\text { 3(ok2734) IV }\end{array}$ & This study & N/A \\
\hline $\begin{array}{l}\text { tomm-20::mCherry II; dkls398 [pie- } \\
1 p:: G F P:: l g g-1+\text { unc-119(+)] }\end{array}$ & This study & $\mathrm{N} / \mathrm{A}$ \\
\hline $\begin{array}{l}\text { daf-2(e1370) III; tomm-20::mCherry II; } \\
\text { dkls398 [pie-1p::GFP::lgg-1 + unc-119(+)] }\end{array}$ & This study & N/A \\
\hline $\begin{array}{l}\text { daf-2(e1370) III; bcls39 V [lim-7p::ced- } \\
1:: \text { GFP + lin-15(+)] }\end{array}$ & This study & N/A \\
\hline
\end{tabular}




\begin{tabular}{|l|l|l|}
\hline daf-2(e1370) III; tomm-20::mCherry II & This study & N/A \\
\hline $\begin{array}{l}\text { daf-2(e1370) III; daf-16(mu86) I; tomm- } \\
\text { 20::mCherry II }\end{array}$ & This study & N/A \\
\hline daf-16(mu86) I; tomm-20::mCherry II & This study & N/A \\
\hline Software and Algorithms & & \\
\hline QuantaSoft ${ }^{\text {IM }}$ & Bio-Rad & $\begin{array}{l}\text { Version } \\
1.7 .4 .0917\end{array}$ \\
\hline Zen & Carl Zeiss Microscopy GmbH & Version 2.0.0.0 \\
\hline Prism 8 for macOS & GraphPad Software, Inc. & Version 8.1.2 \\
\hline ImageJ & Wayne Rasband, NIH & $1.49 \mathrm{v}$ \\
\hline
\end{tabular}

\section{EXPERIMENTAL MODEL AND SUBJECT DETAILS}

\section{Nematode culture}

C. elegans strains used in this study were maintained on $60-\mathrm{mm}$ standard nematode growth medium (NGM) plates seeded with live OP50 E. coli bacteria as a food source, unless otherwise indicated in the METHOD DETAILS below. Nematode strains were incubated at $20^{\circ} \mathrm{C}$ unless otherwise indicated. Age-matched nematodes were used in all experiments with the exception of the multigenerational competition experiment (see below).

\section{METHOD DETAILS}

\section{Nematode lysis}

To prepare nematodes for genotyping and quantification of mtDNA copy number and $\triangle m t D N A$ frequency, nematodes were lysed using the following protocol. Nematodes were transferred to sterile PCR tubes or 96-well PCR plates containing lysis buffer with $100 \mu \mathrm{g} / \mathrm{mL}$ proteinase $\mathrm{K}$. Volume of lysis buffer varied by worm count: $10 \mu \mathrm{L}$ for 
individual adults, pooled larvae, or pooled embryos; $20 \mu \mathrm{L}$ for 5 or 10 pooled adults; 50 $\mu \mathrm{L}$ for pooled nematodes of mixed age (competition experiments, see below). Each tube or plate was then incubated at $-80^{\circ} \mathrm{C}$ for 10 minutes, then at $60^{\circ} \mathrm{C}$ for 60 minutes $(90$ minutes for pooled nematodes), and then at $95^{\circ} \mathrm{C}$ for 15 minutes to inactivate the proteinase K. Nematode lysates were then stored at $-20^{\circ} \mathrm{C}$.

\section{Genetic crosses and genotyping}

To control for nuclear effects on $\triangle$ mtDNA proliferation, hermaphroditic nematodes carrying the $\triangle$ mtDNA allele uaDf5 were serially back-crossed into a male stock of the Bristol (N2) C. elegans nuclear background for six generations. To investigate the role of insulin signaling in selfish mitochondrial genome dynamics, the alleles daf-2(e1370) and daf-16(mu86) were introduced to the $\triangle \mathrm{mtDNA}$ heteroplasmic lineage by classical genetic crosses. To investigate the mechanistic basis by which the insulin signaling pathway regulates mtDNA levels, mutant alleles affecting various putative downstream processes were genetically crossed into the insulin signaling-defective nuclear genotypes. Specifically, the parkin-dependent mitophagy-defective $p d r-1(g k 448)$, the mitochondrial fission-defective $d r p-1(\operatorname{tm} 1108)$, and the apoptosis-defective ced3(ok2734) were each genetically combined with daf-2(e1370), both with and without the daf-16(mu86) allele. Nuclear genotype was confirmed by PCR using the following oligonucleotide primers:

Mutant and wildtype mtDNA:

Exterior forward: 5'-CCATCCGTGCTAGAAGACAA-3' 
Interior forward: 5'-TTGGTGTTACAGGGGCAACA-3'

Reverse: 5'-CTTCTACAGTGCATTGACCTAGTC-3'

daf-2:

Forward: 5'-CATCAAGATCCAGTGCTTCTGAATCGTC-3'

Reverse: 5'-CGGGATGAGACTGTCAAGATTGGAG-3'

daf-16:

Forward: 5'-CACCACGACGCAACACACTAATAGTG-3'

Exterior reverse: 5'-CACGAGACGACGATCCAGGAATCG-3'

Interior reverse: 5'-GGTCTAAACGGAGCAAGTGGTTACTG-3'

$p d r-1:$

Exterior forward: 5'-GAATCATGTTGAAAATGTGACGCGAG-3'

Interior forward: 5'-CTGACACCTGCAACGTAGGTCAAG-3'

Reverse: 5'-GATTTGACTAGAACAGAGGTTGACGAG-3'

$d r p-1:$

Forward: 5'-CGTCGGATCACAGTCGGC-3'

Reverse: 5'-GCACTGACCGCTCTTTCTCC-3'

ced-3:

Exterior forward: 5'-CAGTACTCCTTAAAGGCGCACACC-3'

Interior forward: 5'-GATTGGTCGCAGTTTTCAGTTTAGAGGG-3'

Reverse: 5'-CGATCCCTGTGATGTCTGAAATCCAC-3'

The insulin signaling receptor allele daf-2(e1370) introduces a point mutation that eliminates a $B / p /$ restriction endonuclease recognition site. Following PCR amplification, 
daf-2 PCR products were incubated with Blpl and New England BioLabs CutSmart® buffer at $37^{\circ} \mathrm{C}$ for 2 hours prior to gel electrophoresis. Fluorescent reporters used in this study were genotyped by fluorescence microscopy.

\section{Quantification of mtDNA copy number and $\triangle$ mtDNA frequency}

Quantification of mtDNA copy number and $\triangle \mathrm{mtDNA}$ frequency was accomplished using droplet digital PCR (ddPCR). Nematodes were lysed as described above. Lysates were then diluted in nuclease-free water, with a dilution factor varying depending on nematode concentration: 20x for embryos, 200x for pooled larvae, 200x for single adults, 1000x for pooled adults, 20,000x for pooled nematodes of mixed age from the competition experiments (control diet) or 2,000x for pooled nematodes of mixed age from the competition experiments (restricted diet). The lower dilution factor for the lysates collected from the restricted diet condition was due to the smaller population sizes of nematodes raised on a restricted diet, which arises from reduced fecundity under diet restriction and was reflected in the number of nematodes present in these lysates. Next, either $2 \mu \mathrm{L}$ or $5 \mu \mathrm{L}$ of each dilute nematode lysate was combined with $0.25 \mu \mathrm{L}$ of a $10-\mu \mathrm{M}$ aliquot of each of the following oligonucleotide primers:

For quantifying wildtype mtDNA:

5'-GTCCTTGTGGAATGGTTGAATTTAC-3'

5'-GTACTTAATCACGCTACAGCAGC-3'

For quantifying $\triangle \mathrm{mtDNA}$ :

5-'CCATCCGTGCTAGAAGACAAAG-3' 


\section{5-'CTACAGTGCATTGACCTAGTCATC-3’}

Mixtures of dilute nematode lysate and primer were combined with nuclease-free water and Bio-Rad QX200 ${ }^{\mathrm{TM}}$ ddPCR ${ }^{\mathrm{TM}}$ EvaGreen Supermix to a volume of $25 \mu \mathrm{L}$ in Eppendorf ${ }^{\mathrm{TM}}$ 96-well twin.tec ${ }^{\mathrm{TM}}$ PCR plates. Droplet generation and PCR amplification were performed according to manufacturer protocol with an annealing temperature of $58^{\circ} \mathrm{C}$. For amplification of heteroplasmic nematode lysates, wildtype and $\triangle \mathrm{mtDNA}$ primers were combined in the same reaction, and each droplet was scored as containing either wildtype or mutant template using the 2D amplitude (dual-wavelength) clustering plot option in the Bio-Rad QuantaSoft ${ }^{\mathrm{TM}}$ program.

\section{Respiration assay}

Basal and maximum oxygen consumption rates were measured using the Seahorse XFe96 Analyzer in the High Throughput Screening Facility at Vanderbilt University. One day before experimentation, each well of a 96-well sensor cartridge that comes as part of the Seahorse XFe96 FluxPak was incubated with $200 \mu \mathrm{L}$ of the Seahorse XF Calibrant Solution. On the day of the experiment, 10-20 L4-stage animals were placed into each well of the cell culture microplate. Wells contained either M9 buffer or $10 \mu \mathrm{M}$ FCCP. After calibration, 16 measurements were performed at room temperature. Measurements 12 through 16 were averaged and normalized to number of worms per well.

\section{Fertility}


To assay fertility, day-2 adult nematodes were individually transferred onto NGM plates seeded with live OP50 E. coli and incubated at $20^{\circ} \mathrm{C}$ for 4 hours. The adults were then individually lysed as described above. Fertility was scored as the average number of viable progeny produced per hour during the 4-hour window, where viable progeny were identified as those that had progressed from embryos to larvae within 24 hours of being laid. The $\triangle \mathrm{mtDNA}$ frequency of each parent was determined using ddPCR as described above.

\section{Development}

The impact of $\triangle \mathrm{mtDNA}$ levels on development was assayed by comparing $\triangle \mathrm{mtDNA}$ frequency with developmental stage for each nematode in a population of agesynchronized larvae. To age-synchronize larvae, multiple mature heteroplasmic adults carrying $\triangle \mathrm{mtDNA}$ in the Bristol nuclear background were transferred to an NGM plate seeded with live OP50 E. coli and allowed to lay eggs at $20^{\circ} \mathrm{C}$ for 2 hours. Adults were then removed from the plate. After 48 hours, each nematode was individually lysed and its respective larval stage (L2, L3, or L4) was annotated. None of the nematodes had yet reached adulthood at this point. Embryos that failed to transition to larvae were discarded. The $\triangle \mathrm{mtDNA}$ frequency of each larval nematode was determined using ddPCR as described above.

\section{Sub-organismal selection assay}

Sub-organismal selection for $\triangle \mathrm{mtDNA}$ was quantified by measuring changes in $\triangle \mathrm{mtDNA}$ frequency as a function of developmental stage, and as a function of initial (parental) 
$\triangle m t D N A$ frequency, within a single generation. This was accomplished using two complementary approaches. In the first approach, three individual age-synchronized parents were selected according to initial $\triangle$ mtDNA frequency (parents with low, middle, and high frequency). One age-matched (L4-stage) nematode was selected at random from each line respectively maintained under artificial selection for low $(<50 \%)$, medium $(50-70 \%)$, and high (>70\%) $\Delta$ mtDNA frequency. Each of these nematodes was placed onto a fresh NGM plate seeded with live OP E. coli and incubated for 2 days at $20^{\circ} \mathrm{C}$. Each day-2 adult was then transferred to a fresh food plate every 4 hours and allowed to lay embryos. At each 4-hour time point, approximately one third of the embryos produced were individually lysed. After 12 hours, the adults were individually lysed. A 12-hour time window for embryo production was chosen in order to generate a sufficient offspring count to allow for the establishment of single-brood frequency distributions of $\triangle m t D N A$. The 12-hour time window was divided into 4-hour segments in order to maintain age-synchronicity, as each larva was lysed within 4 hours of being laid across the entire 12-hour period. After 2 days at $20^{\circ} \mathrm{C}$, approximately one third of the L4-stage larvae were individually lysed in the same 4-hour segments to maintain age synchronicity. After an additional 2 days at $20^{\circ} \mathrm{C}$, the remaining one third of offspring were individually lysed in 4-hour segments, as they reached the same age at which their respective parent was lysed. The $\triangle$ mtDNA frequency of each individual was determined using ddPCR as described above and a $\triangle$ mtDNA frequency distribution was generated for each offspring life stage. 
In the second approach, multiple L4-stage heteroplasmic nematodes were selected at random from the stock of nematodes carrying $\triangle$ mtDNA in the Bristol nuclear background. These larvae were transferred to a fresh food plate and incubated for 2 days at $20^{\circ} \mathrm{C}$. The day- 2 adults were then segregated onto individual plates and incubated for 4 hours at $20^{\circ} \mathrm{C}$ to produce age-synchronized progeny. After 4 hours, each parent was individually lysed. Three embryos from each parent were also lysed at the same time, in one pooled lysate per three same-parent embryos. After 2 days, three L4-stage larvae were pooled and lysed from each parent, similar to the lysis of embryos. After another 2 days, three adult progeny were pooled and lysed from each parent as they reached the age at which the parents were lysed. Each parent-progeny lineages was individually segregated from the rest. Since $\triangle$ mtDNA impacts fecundity, the progeny from parents on the lower end of the $\triangle \mathrm{mtDNA}$ frequency are expected to be overrepresented in the offspring sampled from a mixed cohort of parents. Lineages were therefore segregated to ensure that the $\triangle \mathrm{mtDNA}$ frequency from each progeny lysate was being compared with that of its own respective parent, in order to minimize the effect of organism-level selection on $\triangle$ mtDNA. In addition, progeny from each timepoint were lysed in pools of three to reduce the effect of random drift on $\triangle \mathrm{mtDNA}$ frequency. The $\triangle$ mtDNA frequency of parents and each developmental stage of progeny was determined using ddPCR as described above. For the measurement of sub-organismal selection on $\triangle \mathrm{mtDNA}$ under nutrient-variable conditions, each parent was raised from embryo to adult under its respective dietary condition (diet restriction or control). 


\section{Experimental evolution (organismal selection)}

Selection against $\triangle$ mtDNA that occurs strictly at the level of organismal fitness was measured using a competition assay. Heteroplasmic nematodes carrying $\triangle \mathrm{mtDNA}$ in the Bristol nuclear background were combined with Bristol-strain nematodes on $10-\mathrm{cm}$ NGM plates seeded with live OP50 E. coli. For the first generation, heteroplasmic and Bristol strain nematodes were age-synchronized. Age synchronization was accomplished using a bleaching protocol. Nematodes from a mixed-age stock food plate were washed off the plate and into a sterile $1.7 \mathrm{~mL}$ microcentrifuge tube with nuclease-free water. The water was brought to a volume of $750 \mu \mathrm{L}$. The volume of each tube was brought to $1 \mathrm{~mL}$ by adding $100 \mu \mathrm{L}$ of $5 \mathrm{~N} \mathrm{NaOH}$ and $150 \mu \mathrm{L}$ of $6 \%$ bleach. Each nematode tube was incubated at room temperature for 10 minutes with light vortexing every 2 minutes to rupture gravid adults and release embryos. Nematode tubes were centrifuged for 1 minute at $1,000 \mathrm{x} g$ to pellet the nematode embryos. To wash the nematode pellets, the supernatant was removed and replaced with $1 \mathrm{~mL}$ of nuclease-free water. After a second spin for 1 minute at $1,000 \mathrm{x} \mathrm{g}$, the water was removed and the nematode embryos were resuspended in $100 \mu \mathrm{L}$ M9 buffer. The resuspended embryos were then transferred to glass test tubes containing $500 \mu \mathrm{L}$ M9 buffer and incubated overnight at room temperature on a gentle shaker to allow hatching and developmental arrest at the L1 larval stage. On the following day, a glass Pasteur pipette was used to transfer approximately equal quantities of heteroplasmic and homoplasmic-wildtype nematodes onto the $10-\mathrm{cm}$ food plates. Approximately 500 nematodes were transferred to each plate. In addition to 8 competition lines, 8 control 
lines were established by transferring only heteroplasmic nematodes onto food plates, with no homoplasmic-wildtype nematodes to compete against.

Every 3 days, the generation for each experimental line was reset. To do this, nematodes were washed off the plates using sterile M9 buffer into a sterile $1.7 \mathrm{~mL}$ collection tube. Approximately 500 nematodes of mixed age from each line were transferred to a fresh food plate. An additional 500 nematodes were lysed together in a single pooled lysate. Finally, 48 additional adults from each competition line were lysed individually in order to determine the fraction of heteroplasmic nematodes in each competition line at each generational time point. This experiment was continued for 10 consecutive generations.

Experimental evolution was also carried out to quantify nutrient-conditional organismlevel selection. These conditions included 10-cm NGM plates seeded with a restricted or a control diet consisting of UV-killed OP50 E. coli (prepared as described below). Two iterations of this experiment were conducted, one with wildtype nuclear genotype and one with nematodes homozygous for the null daf-16(mu86) allele. Due to the smaller brood sizes among nematodes raised on a restricted diet, 200 nematodes were transferred and another 200 lysed at each generation, instead of the 500 as in the case of the experiment using a live bacterial diet. For these nutrient-conditional competition experiments, 6 replicate lines were propagated for each condition for a total of 8 consecutive generations. Lysis and quantification of $\triangle$ mtDNA frequency by ddPCR were performed as described above. 


\section{Diet restriction}

Diet restriction was accomplished using variable dilutions of UV-inactivated OP50 E. coli bacterial lawns on NGM plates. To prepare diet-restricted food plates, $1 \mathrm{~L}$ of sterile 2xYT liquid microbial growth medium was inoculated with $1 \mathrm{~mL}$ of live OP50 E. coli (suspended in liquid LB) using a sterile serological pipette. The inoculated culture was then incubated overnight on a shaker at $37^{\circ} \mathrm{C}$. The following day, the OP50 E. coli was pelleted by centrifugation for 6 minutes at 3,900 rpm. The pellet was resuspended to a bacterial concentration of approximately $2 \times 10^{10}$ cells $/ \mathrm{mL}$ in sterile M9 buffer. This suspension was seeded onto NGM plates (control) or further diluted 100 -fold to $2 \times 10^{8}$ cells/mL in sterile M9 buffer before being seeded onto NGM plates (diet restriction). Plates were incubated upright at room temperature 4 hours to allow the lawns to dry. To inhibit bacterial growth, plates were irradiated with UV radiation using a Stratagene ${ }^{\circledR}$ UV Stratalinker 1800 set to $9.999 \times 10^{5} \mu \mathrm{J} / \mathrm{cm}^{2}$. To confirm inhibition of bacterial growth, UVtreated plates were incubated overnight at $37^{\circ} \mathrm{C}$.

\section{Insulin signaling inactivation}

Insulin signaling was conditionally inactivated using the allele daf-2(e1370), encoding a temperature-sensitive variant of the $C$. elegans insulin receptor homolog. Because complete loss of insulin signaling during early larval development results in a stage of developmental arrest (dauer), age-synchronized nematodes were incubated at the permissive temperature of $16^{\circ} \mathrm{C}$ until reaching the fourth and final larval stage. L4-stage larvae were then selected at random for either transfer to the restrictive temperature of 
$25^{\circ} \mathrm{C}$ or for continued incubation at $16^{\circ} \mathrm{C}$ as a control. After 4 days of incubation, mature adults were lysed and ddPCR quantification of $\triangle \mathrm{mtDNA}$ frequency was performed as described above. To follow up on the downstream mechanism by which insulin signaling regulates mtDNA dynamics, homoplasmic nematodes were incubated at the restrictive temperature of $25^{\circ} \mathrm{C}$ and mtDNA copy number was measured using the same ddPCR primer pair that was used for quantifying the wildtype mtDNA in heteroplasmic worms.

\section{Knockdown of gene expression}

Expression knockdown of the $C$. elegans insulin signaling receptor homolog, daf-2, was accomplished using feeder plates. Cultures consisting of $2 \mathrm{~mL} \mathrm{LB}$ and $10 \mu \mathrm{L}$ ampicillin were inoculated with a bacterial culture obtained from Source BioScience harboring the Y55D5A_391.b (daf-2) ORF plasmid clone and incubated overnight on a shaker at $37^{\circ} \mathrm{C}$. Bacteria containing the empty plasmid vector were used to establish a control diet. The following day, $750 \mu \mathrm{L}$ of culture was transferred to a flask containing $75 \mathrm{~mL} L B$ and $375 \mu \mathrm{L}$ ampicillin and incubated $4-6$ hours on a shaker at $37^{\circ} \mathrm{C}$, until $\mathrm{OD}_{550-600}>0.8$. An additional $75 \mathrm{~mL}$ LB was added to the culture along with another $375 \mu \mathrm{L}$ ampicillin and $600 \mu \mathrm{L} 1 \mathrm{M}$ isopropyl $\beta$-D-1-thiogalactopyranoside (IPTG) to induce expression of the small interfering RNA. Cultures were incubated another 4 hours on a shaker at $37^{\circ} \mathrm{C}$. Cultures were then centrifuged for 6 minutes at $3,900 \mathrm{rpm}$ and the resulting bacterial pellets were each resuspended in $6 \mathrm{~mL}$ M9 buffer with $8 \mathrm{mM}$ IPTG. Next, 250 $\mu \mathrm{L}$ of resuspension was seeded onto each NGM plate. Plates were allowed to dry at room temperature in the dark and then stored at $4^{\circ} \mathrm{C}$ until use. Synchronized L4-stage nematodes were transferred at random to either RNAi knockdown or control plates and 
incubated at $25^{\circ} \mathrm{C}$ until day 4 of adulthood to match the conditions that were used for the daf-2 mutant allele. Day-4 adults were lysed and their mtDNA copy number was quantified using ddPCR as described above.

\section{Live imaging}

Overall mitochondrial content across the wildtype and defective insulin signaling genotypes was measured using the mitochondrial reporter TOMM-20::mCherry. Agesynchronized nematodes were incubated for 2 days from the L4 stage to mature adulthood at $25^{\circ} \mathrm{C}$, immobilized with $10 \mathrm{mM}$ levamisole, and placed on the center of a $2 \%$ agarose pad on a microscope slide. Nematodes were imaged at $10 x$ magnification using a Leica DM6000 B compound fluorescence microscope and mitochondrial fluorescence was quantified using ImageJ. Apoptosis was imaged in daf-2(e1370) mutant nematodes and wildtype controls using the CED-1::GFP reporter. Agesynchronized nematodes were incubated for 2 days from the L4 stage to mature adulthood at $25^{\circ} \mathrm{C}$ before being immobilized and mounted on microscope slides as described above. Apoptotic cells were imaged using a Zeiss LSM 880 Confocal Laser Scanning microscope at 20x magnification.

\section{Staining and imaging of germline nuclei}

Nematode germline nuclei were quantified across age-synchronized mature adults homozygous for daf-2(e1370) or daf-16(mu86), as well as in double-mutants and wildtype controls. For each genotype, age-synchronized L4-stage nematodes were incubated for 2 days at $25^{\circ} \mathrm{C}$ and then placed in a plate containing $3 \mathrm{~mL}$ of PBS with 
$200 \mu \mathrm{M}$ levamisole. To dissect the nematode gonads, each adult was decapitated using two 25Gx1" hypodermic needles in a scissor-motion under a dissecting microscope. Dissected gonads were fixed for 20 minutes in $3 \%$ paraformaldehyde. Fixed gonads were transferred to a glass test tube using a glass Pasteur pipette and the paraformaldehyde was replaced with PBT (PBS buffer with $0.1 \%$ Tween 20) and incubated for 15 minutes at room temperature. The PBT was then replaced with PBT containing $100 \mathrm{ng} / \mathrm{mL}$ 4',6'-diamidino-2-phenylindole dihydrochloride (DAPI) and the gonads were incubated in darkness for another 15 minutes at room temperature. Gonads were then subjected to $3 x$ consecutive washes, each consisting of a 1-minute centrifugation at $1,000 \mathrm{rpm}$ followed by replacement of the PBT. Gonads were then mounted directly onto a $2 \%$ agarose pad on the center of a microscope slide and imaged using a Zeiss LSM 880 Confocal Laser Scanning microscope at 20x magnification.

Ahier, A., Dai, C.Y., Tweedie, A., Bezawork-Geleta, A., Kirmes, I., and Zuryn, S. (2018). Affinity purification of cell-specific mitochondria from whole animals resolves patterns of genetic mosaicism. Nat Cell Biol 20, 352-360.

Angelo, G., and Van Gilst, M.R. (2009). Starvation protects germline stem cells and extends reproductive longevity in C. elegans. Science 326, 954-958.

Badisco, L., Van Wielendaele, P., and Vanden Broeck, J. (2013). Eat to reproduce: a key role for the insulin signaling pathway in adult insects. Front Physiol 4, 202.

Bratic, I., Hench, J., Henriksson, J., Antebi, A., Burglin, T.R., and Trifunovic, A. (2009). Mitochondrial DNA level, but not active replicase, is essential for Caenorhabditis elegans development. Nucleic Acids Res 37, 1817-1828.

Cao, P., Dey, A., Vassallo, C.N., and Wall, D. (2015). How Myxobacteria Cooperate. J Mol Biol 427, 3709-3721.

Chatre, L., and Ricchetti, M. (2013). Prevalent coordination of mitochondrial DNA transcription and initiation of replication with the cell cycle. Nucleic Acids Res 41, 30683078.

Chen, X., and Perc, M. (2014). Excessive abundance of common resources deters social responsibility. Sci Rep 4, 4161. 
Chisholm, R.L., and Firtel, R.A. (2004). Insights into morphogenesis from a simple developmental system. Nat Rev Mol Cell Biol 5, 531-541.

Clark, K.A., Howe, D.K., Gafner, K., Kusuma, D., Ping, S., Estes, S., and Denver, D.R. (2012). Selfish little circles: transmission bias and evolution of large deletion-bearing mitochondrial DNA in Caenorhabditis briggsae nematodes. PLoS One 7, e41433.

Danielsen, E.T., Moeller, M.E., and Rewitz, K.F. (2013). Nutrient signaling and developmental timing of maturation. Curr Top Dev Biol 105, 37-67.

Das, D., and Arur, S. (2017). Conserved insulin signaling in the regulation of oocyte growth, development, and maturation. Mol Reprod Dev 84, 444-459.

de Vargas Roditi, L., Boyle, K.E., and Xavier, J.B. (2013). Multilevel selection analysis of a microbial social trait. Mol Syst Biol 9, 684.

Depuydt, G., Xie, F., Petyuk, V.A., Smolders, A., Brewer, H.M., Camp, D.G., 2nd, Smith, R.D., and Braeckman, B.P. (2014). LC-MS proteomics analysis of the insulin/IGF-1-deficient Caenorhabditis elegans daf-2(e1370) mutant reveals extensive restructuring of intermediary metabolism. J Proteome Res 13, 1938-1956.

Dobata, S., Sasaki, T., Mori, H., Hasegawa, E., Shimada, M., and Tsuji, K. (2009). Cheater genotypes in the parthenogenetic ant Pristomyrmex punctatus. Proc Biol Sci 276, 567-574.

Drummond-Barbosa, D., and Spradling, A.C. (2001). Stem cells and their progeny respond to nutritional changes during Drosophila oogenesis. Dev Biol 231, 265-278.

Dubie, J.J., Caraway, A.R., Stout, M.M., Katju, V., and Bergthorsson, U. (2020). The conflict within: origin, proliferation and persistence of a spontaneously arising selfish mitochondrial genome. Philos Trans R Soc Lond B Biol Sci 375, 20190174.

Ducasse, H., Arnal, A., Vittecoq, M., Daoust, S.P., Ujvari, B., Jacqueline, C., Tissot, T., Ewald, P., Gatenby, R.A., King, K.C., et al. (2015). Cancer: an emergent property of disturbed resourcerich environments? Ecology meets personalized medicine. Evol Appl 8, 527-540.

Fisher, R.M., and Regenberg, B. (2019). Multicellular group formation in Saccharomyces cerevisiae. Proc Biol Sci 286, 20191098.

Frank, S.A. (1997). The Price Equation, Fisher's fundamental theorem, kin selection, and causal analysis. Evolution 51, 1712-1729.

Gaillard, C., and Bernardi, G. (1979). Nucleotide-Sequence of the Mitochondrial Genome of a Spontaneous Petite Mutant of Yeast. Mol Gen Genet 174, 335-337.

Ghoul, M., Griffin, A.S., and West, S.A. (2014). Toward an evolutionary definition of cheating. Evolution 68, 318-331.

Gitschlag, B.L., Kirby, C.S., Samuels, D.C., Gangula, R.D., Mallal, S.A., and Patel, M.R. (2016). Homeostatic Responses Regulate Selfish Mitochondrial Genome Dynamics in C. elegans. Cell Metab 24, 91-103.

Goldring, E.S., Grossman, L.I., and Marmur, J. (1971). Petite mutation in yeast. II. Isolation of mutants containing mitochondrial deoxyribonucleic acid of reduced size. J Bacteriol 107, 377-381.

Gottlieb, S., and Ruvkun, G. (1994). daf-2, daf-16 and daf-23: genetically interacting genes controlling Dauer formation in Caenorhabditis elegans. Genetics 137, 107-120.

Greer, E.L., Dowlatshahi, D., Banko, M.R., Villen, J., Hoang, K., Blanchard, D., Gygi, S.P., and Brunet, A. (2007). An AMPK-FOXO pathway mediates longevity induced by a novel method of dietary restriction in C. elegans. Curr Biol 17, 1646-1656.

Gulli, J.G., Herron, M.D., and Ratcliff, W.C. (2019). Evolution of altruistic cooperation among nascent multicellular organisms. Evolution 73, 1012-1024. 
Han, J.J., Zhang, L., Guo, H., Wysham, W.Z., Roque, D.R., Willson, A.K., Sheng, X.G., Zhou, C.X., and Bae-Jump, V.L. (2015). Glucose promotes cell proliferation, glucose uptake and invasion in endometrial cancer cells via AMPK/mTOR/S6 and MAPK signaling. Gynecol Oncol 138, 668-675.

Hibshman, J.D., Doan, A.E., Moore, B.T., Kaplan, R.E., Hung, A., Webster, A.K., Bhatt, D.P., Chitrakar, R., Hirschey, M.D., and Baugh, L.R. (2017). daf-16/FoxO promotes gluconeogenesis and trehalose synthesis during starvation to support survival. Elife 6 .

Hill, J.H., Chen, Z., and Xu, H. (2014). Selective propagation of functional mitochondrial DNA during oogenesis restricts the transmission of a deleterious mitochondrial variant. Nat Genet 46, 389-392.

Hollis, B. (2012). Rapid antagonistic coevolution between strains of the social amoeba Dictyostelium discoideum. Proc Biol Sci 279, 3565-3571.

Holt, I.J., Speijer, D., and Kirkwood, T.B. (2014). The road to rack and ruin: selecting deleterious mitochondrial DNA variants. Philos Trans R Soc Lond B Biol Sci 369, 20130451. Howe, D.K., and Denver, D.R. (2008). Muller's Ratchet and compensatory mutation in Caenorhabditis briggsae mitochondrial genome evolution. BMC Evol Biol 8, 62.

Hsieh, E.A., Chai, C.M., and Hellerstein, M.K. (2005). Effects of caloric restriction on cell proliferation in several tissues in mice: role of intermittent feeding. Am J Physiol-Endoc M 288, E965-E972.

Khare, A., Santorelli, L.A., Strassmann, J.E., Queller, D.C., Kuspa, A., and Shaulsky, G. (2009). Cheater-resistance is not futile. Nature 461, 980-982.

Klotz, L.O., Sanchez-Ramos, C., Prieto-Arroyo, I., Urbanek, P., Steinbrenner, H., and Monsalve, M. (2015). Redox regulation of FoxO transcription factors. Redox Biol 6, 51-72.

Klucnika, A., and Ma, H. (2019). A battle for transmission: the cooperative and selfish animal mitochondrial genomes. Open Biol 9, 180267.

Koschwanez, J.H., Foster, K.R., and Murray, A.W. (2013). Improved use of a public good selects for the evolution of undifferentiated multicellularity. Elife 2, e00367.

Kramer, J.M., Slade, J.D., and Staveley, B.E. (2008). foxo is required for resistance to amino acid starvation in Drosophila. Genome 51, 668-672.

Lee, S., and Dong, H.H. (2017). FoxO integration of insulin signaling with glucose and lipid metabolism. J Endocrinol 233, R67-R79.

Letellier, T., Heinrich, R., Malgat, M., and Mazat, J.P. (1994). The kinetic basis of threshold effects observed in mitochondrial diseases: a systemic approach. Biochem J 302 ( Pt 1), 171-174.

Li, S.I., and Purugganan, M.D. (2011). The cooperative amoeba: Dictyostelium as a model for social evolution. Trends Genet 27, 48-54.

Liau, W.S., Gonzalez-Serricchio, A.S., Deshommes, C., Chin, K., and LaMunyon, C.W. (2007). A persistent mitochondrial deletion reduces fitness and sperm performance in heteroplasmic populations of C. elegans. BMC Genet 8,8 .

Lieber, T., Jeedigunta, S.P., Palozzi, J.M., Lehmann, R., and Hurd, T.R. (2019). Mitochondrial fragmentation drives selective removal of deleterious mtDNA in the germline. Nature 570, 380-384.

Lin, Y.F., Schulz, A.M., Pellegrino, M.W., Lu, Y., Shaham, S., and Haynes, C.M. (2016). Maintenance and propagation of a deleterious mitochondrial genome by the mitochondrial unfolded protein response. Nature 533, 416-419. 
Lopez, A.L., 3rd, Chen, J., Joo, H.J., Drake, M., Shidate, M., Kseib, C., and Arur, S. (2013). DAF-2 and ERK couple nutrient availability to meiotic progression during Caenorhabditis elegans oogenesis. Dev Cell 27, 227-240.

Ma, H., and O'Farrell, P.H. (2016). Selfish drive can trump function when animal mitochondrial genomes compete. Nat Genet 48, 798-802.

$\mathrm{Ma}, \mathrm{H} ., \mathrm{Xu}, \mathrm{H}$. , and O'Farrell, P.H. (2014). Transmission of mitochondrial mutations and action of purifying selection in Drosophila melanogaster. Nat Genet 46, 393-397.

MacAlpine, D.M., Kolesar, J., Okamoto, K., Butow, R.A., and Perlman, P.S. (2001). Replication and preferential inheritance of hypersuppressive petite mitochondrial DNA. EMBO J 20, 1807-1817.

Martins, R., Lithgow, G.J., and Link, W. (2016). Long live FOXO: unraveling the role of FOXO proteins in aging and longevity. Aging Cell 15, 196-207.

Michaelson, D., Korta, D.Z., Capua, Y., and Hubbard, E.J.A. (2010). Insulin signaling promotes germline proliferation in C. elegans. Development 137, 671-680.

Michod, R.E., Viossat, Y., Solari, C.A., Hurand, M., and Nedelcu, A.M. (2006). Life-history evolution and the origin of multicellularity. J Theor Biol 239, 257-272.

Murphy, C.T., McCarroll, S.A., Bargmann, C.I., Fraser, A., Kamath, R.S., Ahringer, J., Li, H., and Kenyon, C. (2003). Genes that act downstream of DAF-16 to influence the lifespan of Caenorhabditis elegans. Nature 424, 277-283.

Narbonne, P., and Roy, R. (2006). Regulation of germline stem cell proliferation downstream of nutrient sensing. Cell Div 1.

Narita, S., Nara, T., Sato, H., Koizumi, A., Huang, M., Inoue, T., and Habuchi, T. (2019). Research Evidence on High-Fat Diet-Induced Prostate Cancer Development and Progression. J Clin Med 8.

Newlon, C.S., and Fangman, W.L. (1975). Mitochondrial DNA synthesis in cell cycle mutants of Saccharomyces cerevisiae. Cell 5, 423-428.

O-Sullivan, I., Zhang, W.W., Wasserman, D.H., Liew, C.W., Liu, J., Paik, J., DePinho, R.A., Stolz, D.B., Kahn, C.R., Schwartz, M.W., et al. (2015). Fox01 integrates direct and indirect effects of insulin on hepatic glucose production and glucose utilization (vol 6, 7079, 2015). Nat Commun 6.

O'Brien, S., Lujan, A.M., Paterson, S., Cant, M.A., and Buckling, A. (2017). Adaptation to public goods cheats in Pseudomonas aeruginosa. Proc Biol Sci 284.

Ozkaya, O., Xavier, K.B., Dionisio, F., and Balbontin, R. (2017). Maintenance of Microbial Cooperation Mediated by Public Goods in Single- and Multiple-Trait Scenarios. J Bacteriol 199.

Pazdernik, N., and Schedl, T. (2013). Introduction to germ cell development in Caenorhabditis elegans. Adv Exp Med Biol 757, 1-16.

Pereda, M., Zurro, D., Santos, J.I., Briz, I.G.I., Alvarez, M., Caro, J., and Galan, J.M. (2017). Emergence and Evolution of Cooperation Under Resource Pressure. Sci Rep 7, 45574.

Picard, M., Zhang, J., Hancock, S., Derbeneva, O., Golhar, R., Golik, P., O'Hearn, S., Levy, S., Potluri, P., Lvova, M., et al. (2014). Progressive increase in mtDNA 3243A>G heteroplasmy causes abrupt transcriptional reprogramming. Proc Natl Acad Sci U S A 111, E4033-4042.

Porte, D., Baskin, D.G., and Schwartz, M.W. (2005). Perspectives in diabetes - Insulin signaling in the central nervous system - A critical role in metabolic homeostasis and disease from C-elegans to humans. Diabetes 54, 1264-1276. 
Price, G.R. (1972). Extension of Covariance Selection Mathematics. Ann Hum Genet 35, 485\&.

Puig, 0., and Tjian, R. (2006). Nutrient availability and growth: regulation of insulin signaling by dFOXO/FOX01. Cell Cycle 5, 503-505.

Rollins, L.A., Woolnough, A.P., Fanson, B.G., Cummins, M.L., Crowley, T.M., Wilton, A.N., Sinclair, R., Butler, A., and Sherwin, W.B. (2016). Selection on Mitochondrial Variants Occurs between and within Individuals in an Expanding Invasion. Mol Biol Evol 33, 9951007.

Sciacco, M., Bonilla, E., Schon, E.A., DiMauro, S., and Moraes, C.T. (1994). Distribution of wild-type and common deletion forms of mtDNA in normal and respiration-deficient muscle fibers from patients with mitochondrial myopathy. Hum Mol Genet 3, 13-19.

Sena, E.P., Welch, J.W., Halvorson, H.O., and Fogel, S. (1975). Nuclear and mitochondrial deoxyribonucleic acid replication during mitosis in Saccharomyces cerevisiae. J Bacteriol 123, 497-504.

Shidara, Y., Yamagata, K., Kanamori, T., Nakano, K., Kwong, J.Q., Manfredi, G., Oda, H., and Ohta, S. (2005). Positive contribution of pathogenic mutations in the mitochondrial genome to the promotion of cancer by prevention from apoptosis. Cancer Res 65, 1655-1663.

Shim, Y.H., Chun, J.H., Lee, E.Y., and Paik, Y.K. (2002). Role of cholesterol in germ-line development of Caenorhabditis elegans. Mol Reprod Dev 61, 358-366.

Shiojima, I., Yefremashvili, M., Luo, Z., Kureishi, Y., Takahashi, A., Tao, J., Rosenzweig, A., Kahn, C.R., Abel, E.D., and Walsh, K. (2002). Akt signaling mediates postnatal heart growth in response to insulin and nutritional status. J Biol Chem 277, 37670-37677.

Shou, W. (2015). Acknowledging selection at sub-organismal levels resolves controversy on pro-cooperation mechanisms. Elife 4.

Stewart, J.B., Freyer, C., Elson, J.L., Wredenberg, A., Cansu, Z., Trifunovic, A., and Larsson, N.G. (2008). Strong purifying selection in transmission of mammalian mitochondrial DNA. PLoS Biol 6, e10.

Strassmann, J.E., Zhu, Y., and Queller, D.C. (2000). Altruism and social cheating in the social amoeba Dictyostelium discoideum. Nature 408, 965-967.

Takakuwa, T., Nakashima, Y., Koh, H., Nakane, T., Nakamae, H., and Hino, M. (2019). ShortTerm Fasting Induces Cell Cycle Arrest in Immature Hematopoietic Cells and Increases the Number of Naive T Cells in the Bone Marrow of Mice. Acta Haematol-Basel 141, 189-198.

Taylor, D.R., Zeyl, C., and Cooke, E. (2002). Conflicting levels of selection in the accumulation of mitochondrial defects in Saccharomyces cerevisiae. Proc Natl Acad Sci U S A 99, 3690-3694.

Teleman, A.A., Hietakangas, V., Sayadian, A.C., and Cohen, S.M. (2008). Nutritional control of protein biosynthetic capacity by insulin via Myc in Drosophila. Cell Metab 7, 21-32.

Tepper, R.G., Ashraf, J., Kaletsky, R., Kleemann, G., Murphy, C.T., and Bussemaker, H.J. (2013). PQM-1 complements DAF-16 as a key transcriptional regulator of DAF-2-mediated development and longevity. Cell 154, 676-690.

Tilokani, L., Nagashima, S., Paupe, V., and Prudent, J. (2018). Mitochondrial dynamics: overview of molecular mechanisms. Essays Biochem 62, 341-360.

Tsang, W.Y., and Lemire, B.D. (2002). Stable heteroplasmy but differential inheritance of a large mitochondrial DNA deletion in nematodes. Biochem Cell Biol 80, 645-654.

Tucci, P. (2012). Caloric restriction: is mammalian life extension linked to p53? Aging-Us 4, 525-534. 
Vanthournout, B., Greve, M., Bruun, A., Bechsgaard, J., Overgaard, J., and Bilde, T. (2016). Benefits of Group Living Include Increased Feeding Efficiency and Lower Mass Loss during Desiccation in the Social and Inbreeding Spider Stegodyphus dumicola. Front Physiol 7, 18. Wang, Z.H., Liu, Y., Chaitankar, V., Pirooznia, M., and Xu, H. (2019). Electron transport chain biogenesis activated by a JNK-insulin-Myc relay primes mitochondrial inheritance in Drosophila. Elife 8.

Webb, A.E., Kundaje, A., and Brunet, A. (2016). Characterization of the direct targets of FOXO transcription factors throughout evolution. Aging Cell 15, 673-685.

West, S.A., Fisher, R.M., Gardner, A., and Kiers, E.T. (2015). Major evolutionary transitions in individuality. Proc Natl Acad Sci U S A 112, 10112-10119.

Wilson, D.S., and Wilson, E.O. (2007). Rethinking the theoretical foundation of sociobiology. Q Rev Biol 82, 327-348.

Win, M.T., Yamamoto, Y., Munesue, S., Han, D., Harada, S., and Yamamoto, H. (2013). Validated Liquid Culture Monitoring System for Lifespan Extension of Caenorhabditis elegans through Genetic and Dietary Manipulations. Aging Dis 4, 178-185.

Zhang, Y., Wang, Z.H., Liu, Y., Chen, Y., Sun, N., Gucek, M., Zhang, F., and Xu, H. (2019). PINK1 Inhibits Local Protein Synthesis to Limit Transmission of Deleterious Mitochondrial DNA Mutations. Mol Cell 73, 1127-1137 e1125. 
bioRxiv preprint doi: https://doi.org/10.1101/2020.01.30.927202; this version posted January 31, 2020. The copyright holder for this preprint (which was not certified by peer review) is the author/funder, who has granted bioRxiv a license to display the preprint in perpetuity. It is made available under aCC-BY-NC-ND 4.0 International license.

Figure 1

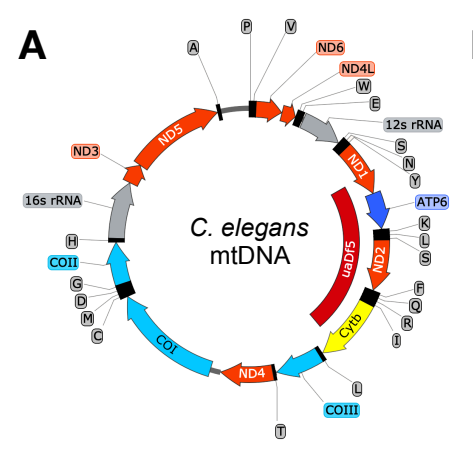

C

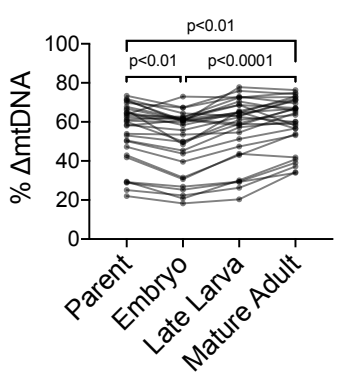

B
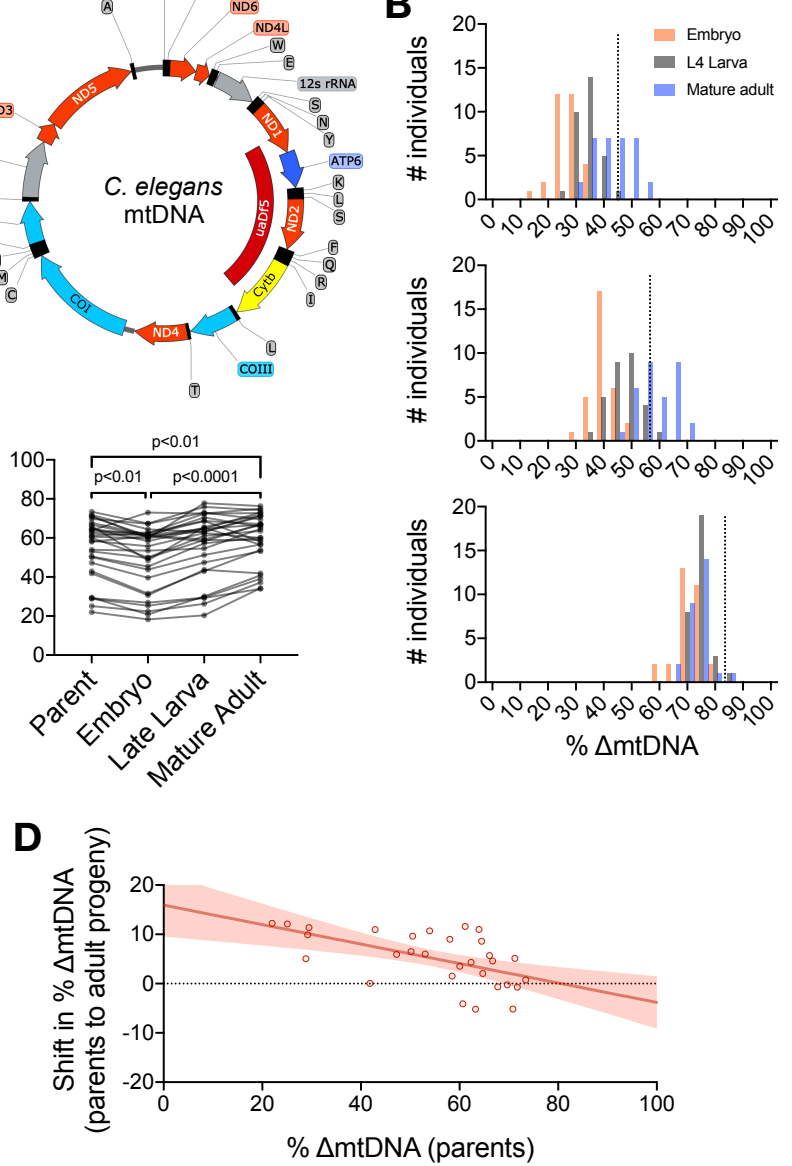
bioRxiv preprint doi: https://doi.org/10.1101/2020.01.30.927202; this version posted January 31, 2020. The copyright holder for this preprint (which was not certified by peer review) is the author/funder, who has granted bioRxiv a license to display the preprint in perpetuity. It is made available under aCC-BY-NC-ND 4.0 International license.

Figure 2
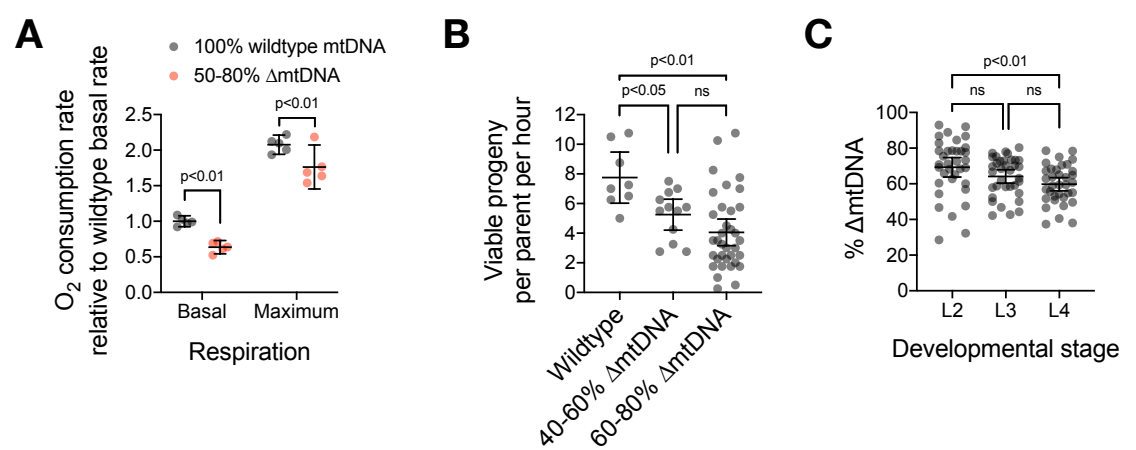

D

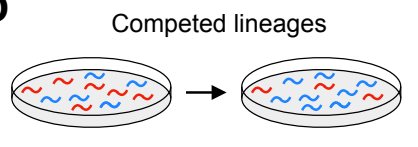

Non-competed lineages

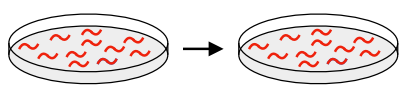

$\sim$ Wildtype individuals

$\sim$ Carriers of $\triangle \mathrm{mtDNA}$
$\mathbf{E}$

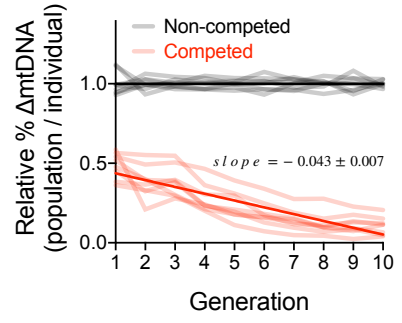


bioRxiv preprint doi: https://doi.org/10.1101/2020.01.30.927202; this version posted January 31, 2020. The copyright holder for this preprint (which was not certified by peer review) is the author/funder, who has granted bioRxiv a license to display the preprint in perpetuity. It is made available under aCC-BY-NC-ND 4.0 International license.

Figure 3

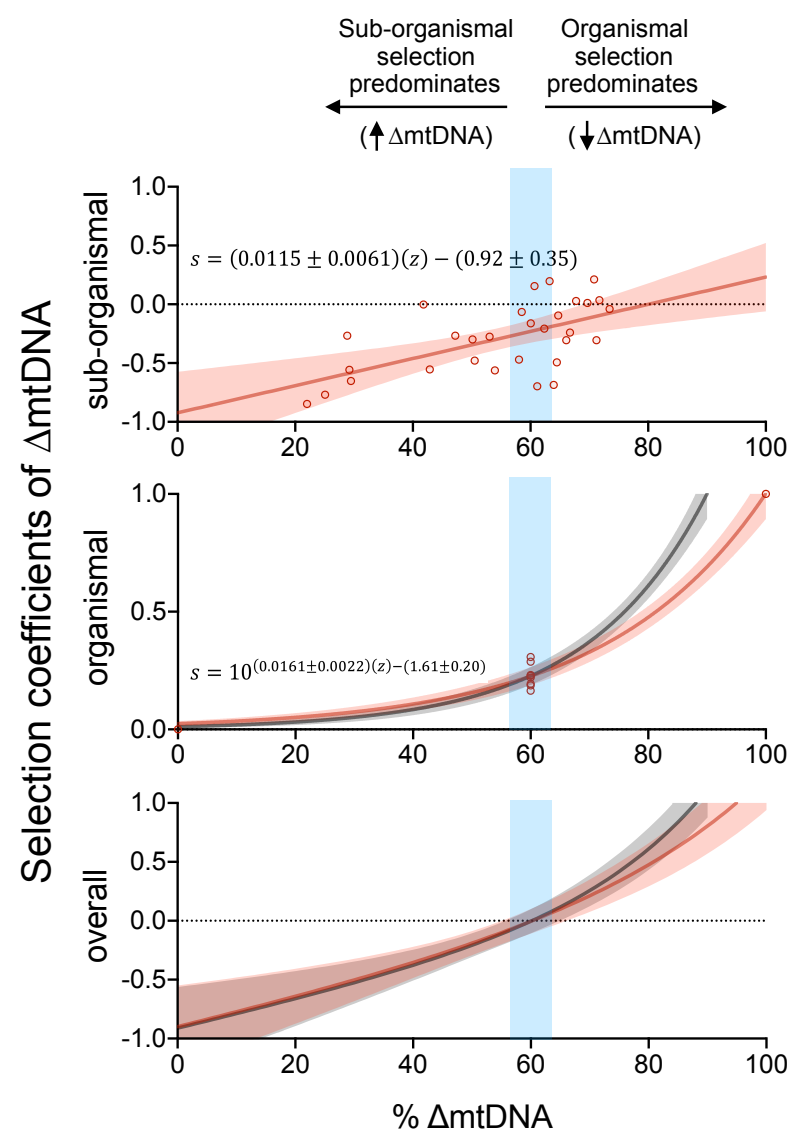


bioRxiv preprint doi: https://doi.org/10.1101/2020.01.30.927202; this version posted January 31, 2020. The copyright holder for this preprint (which was not certified by peer review) is the author/funder, who has granted bioRxiv a license to display the preprint in perpetuity. It is made available under aCC-BY-NC-ND 4.0 International license.

Figure 4
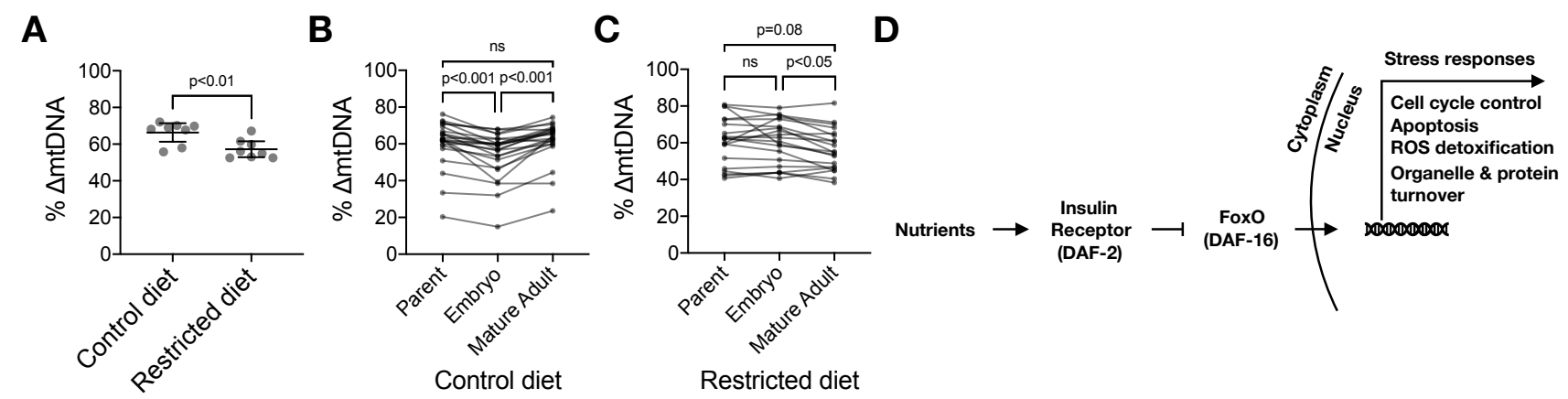

Restricted diet

E

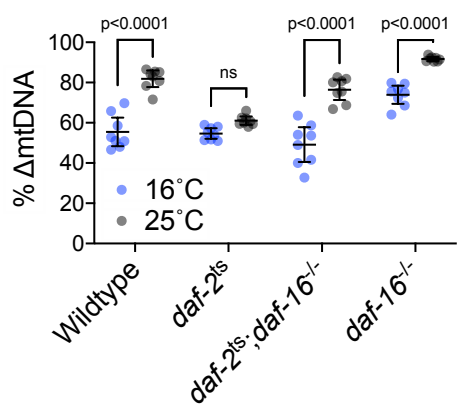

$\mathbf{F}$

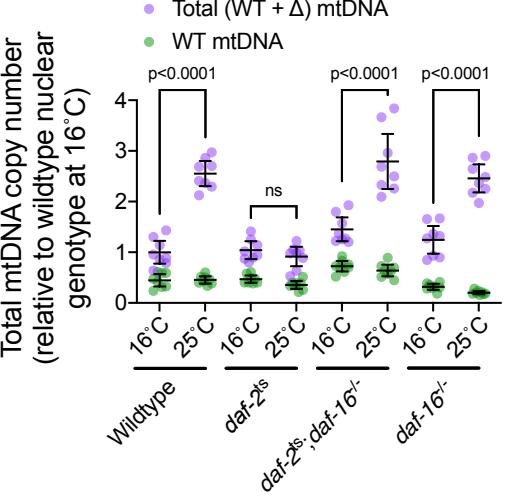

G

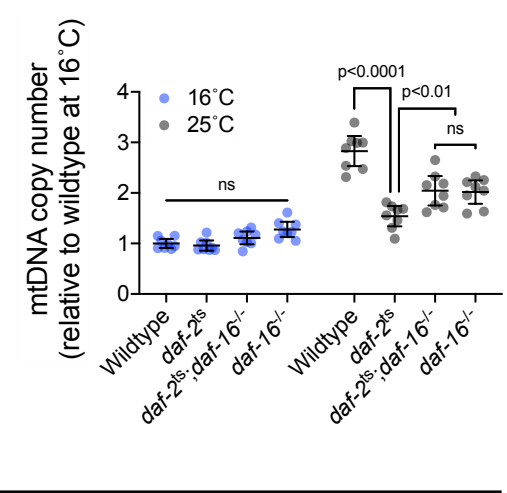

Temperature and nuclear genotype 
bioRxiv preprint doi: https://doi.org/10.1101/2020.01.30.927202; this version posted January 31, 2020. The copyright holder for this preprint (which was not certified by peer review) is the author/funder, who has granted bioRxiv a license to display the preprint in perpetuity. It is made available under aCC-BY-NC-ND 4.0 International license.

Figure 5
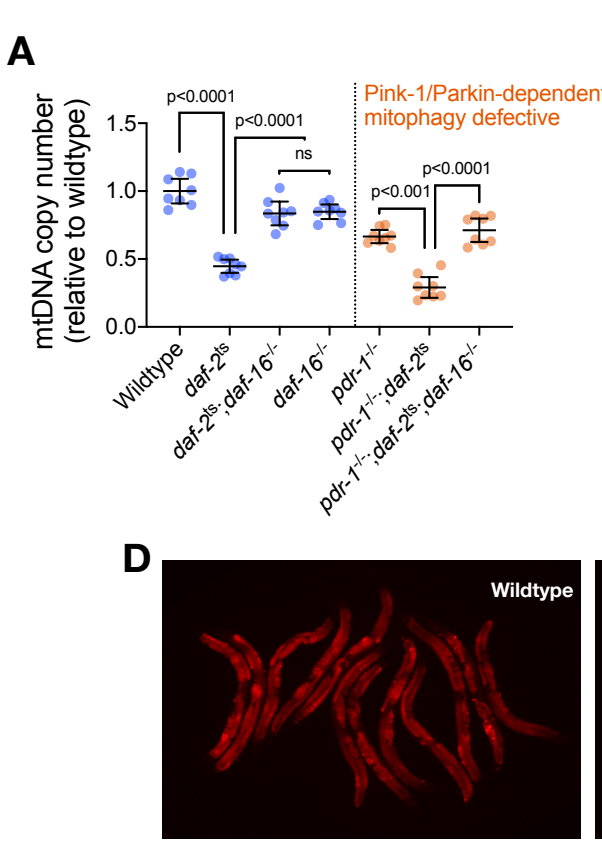

B
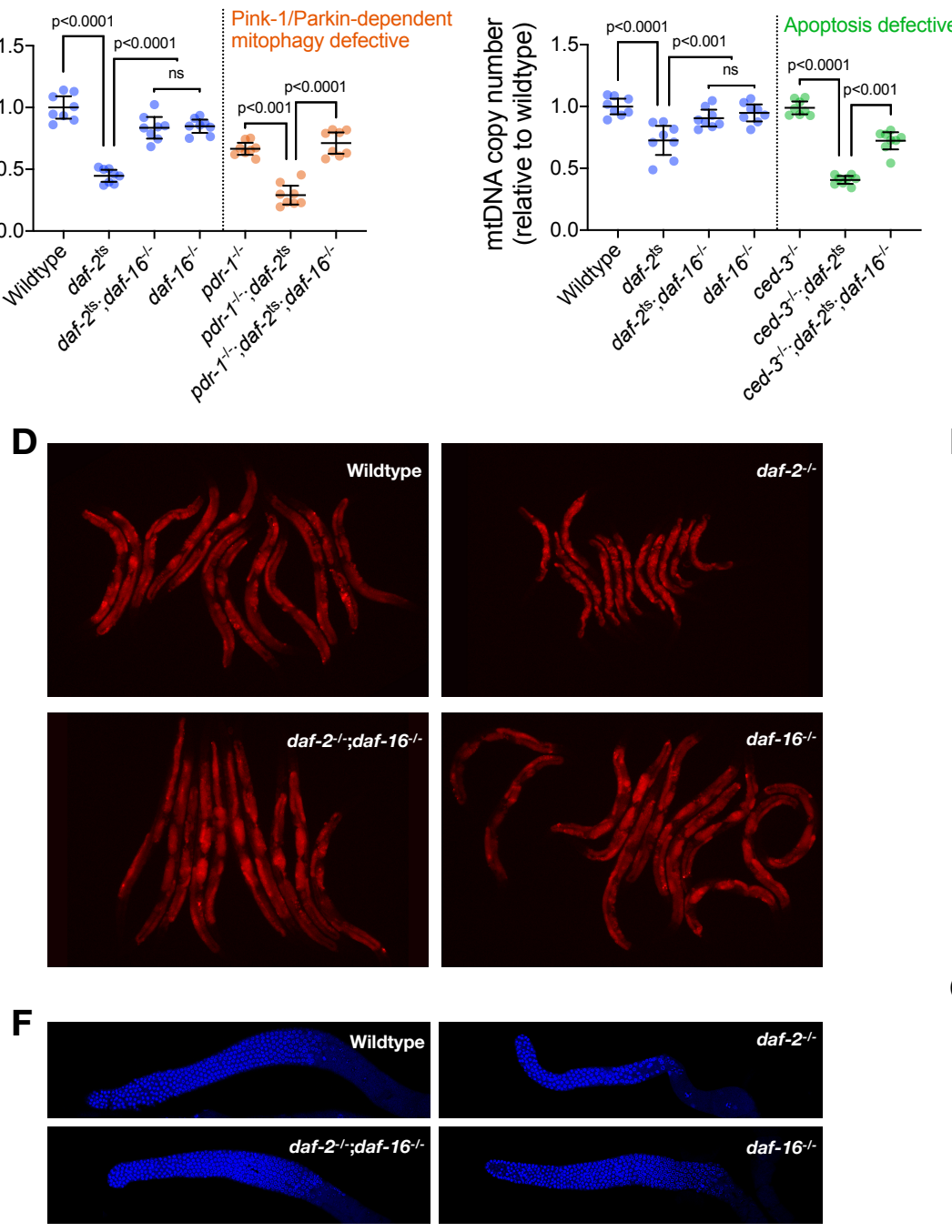

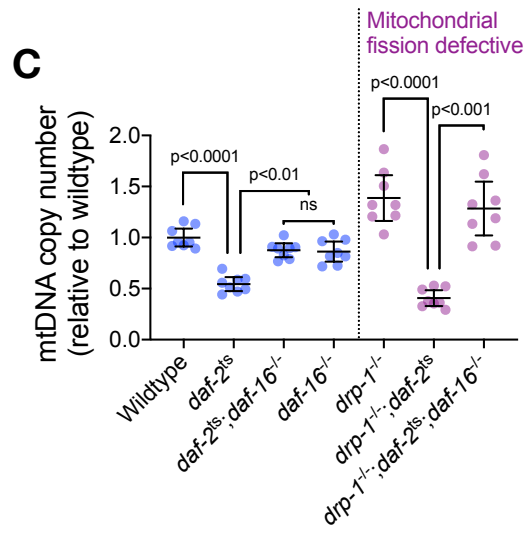

E
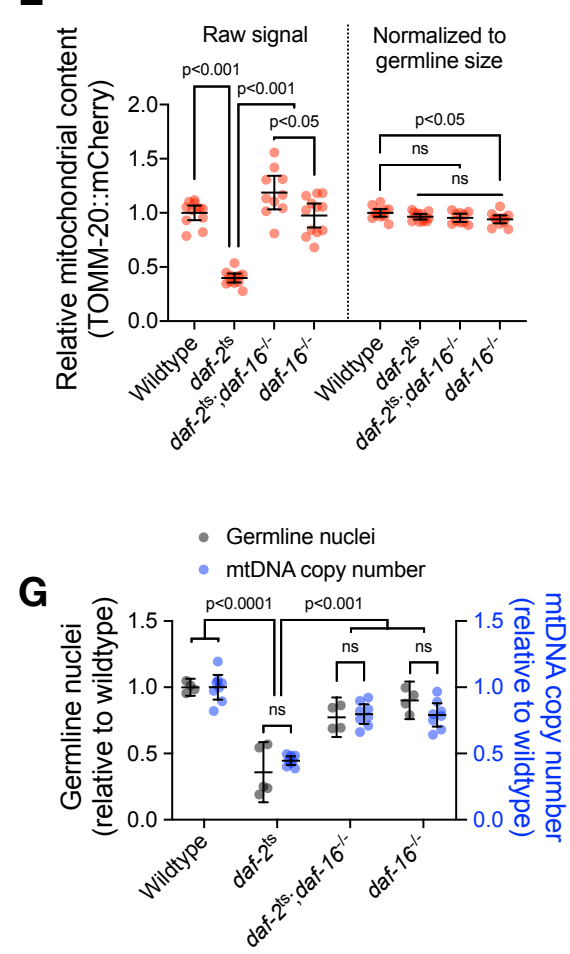
bioRxiv preprint doi: https://doi.org/10.1101/2020.01.30.927202; this version posted January 31, 2020. The copyright holder for this preprint (which was not certified by peer review) is the author/funder, who has granted bioRxiv a license to display the preprint in perpetuity. It is made available under aCC-BY-NC-ND 4.0 International license.

Figure 6

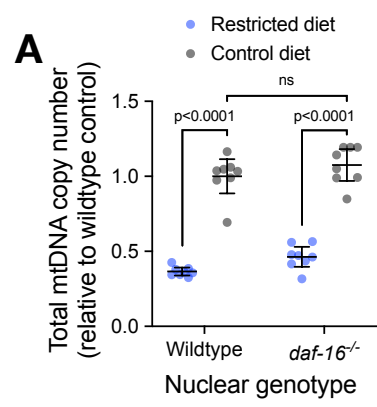

C

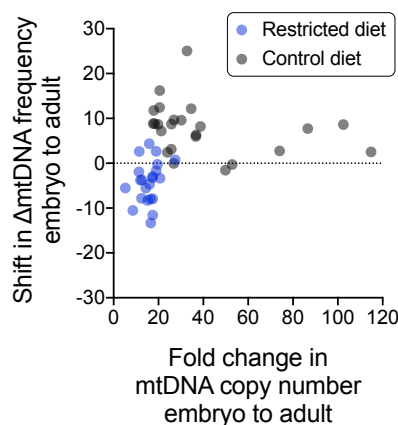

B

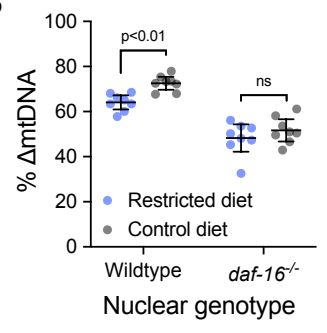

D

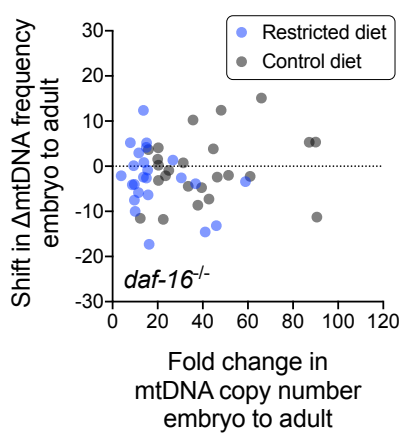


bioRxiv preprint doi: https://doi.org/10.1101/2020.01.30.927202; this version posted January 31, 2020. The copyright holder for this preprint (which was not certified by peer review) is the author/funder, who has granted bioRxiv a license to display the preprint in perpetuity. It is made available under aCC-BY-NC-ND 4.0 International license.

Figure 7
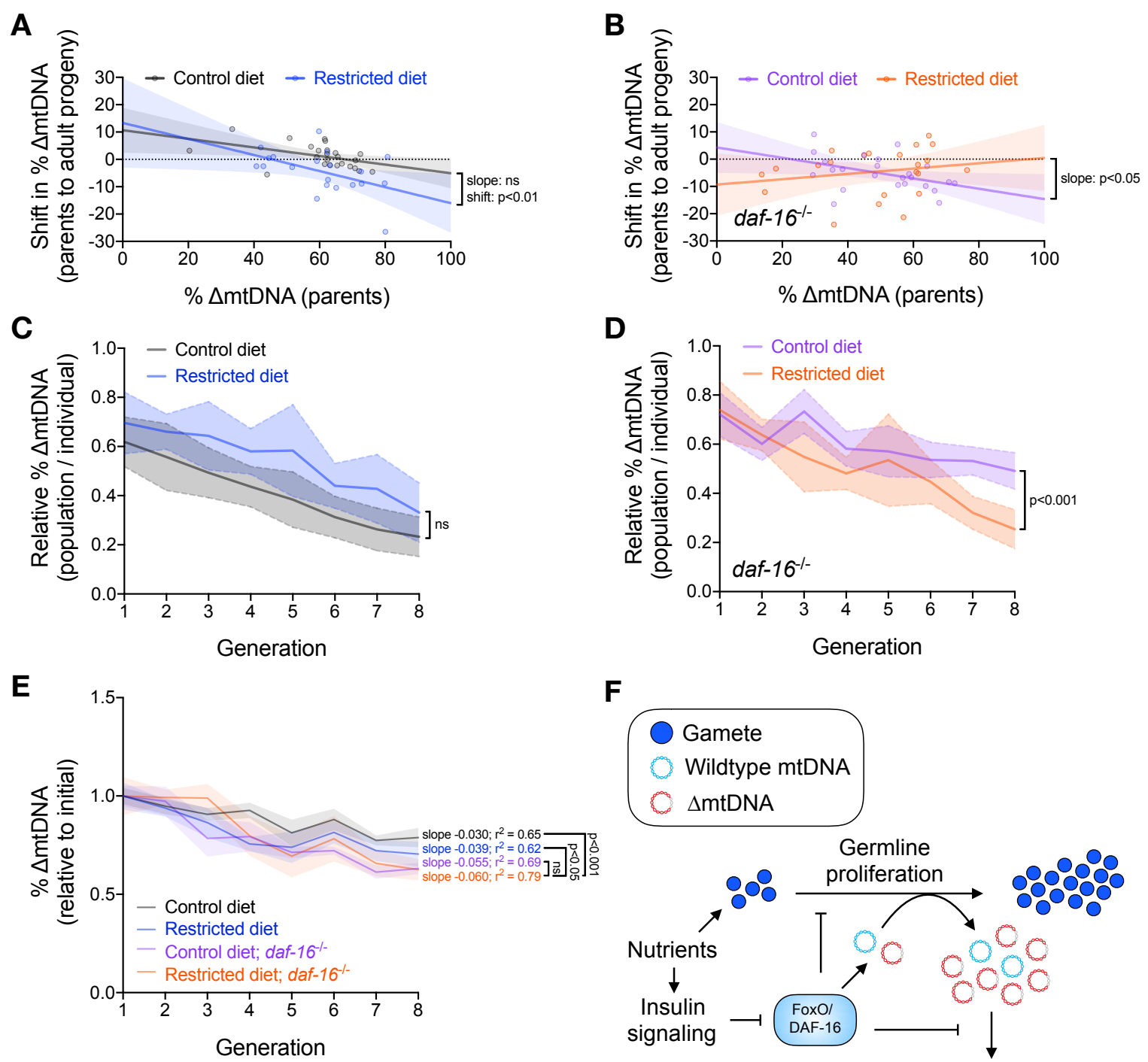

$\mathbf{F}$

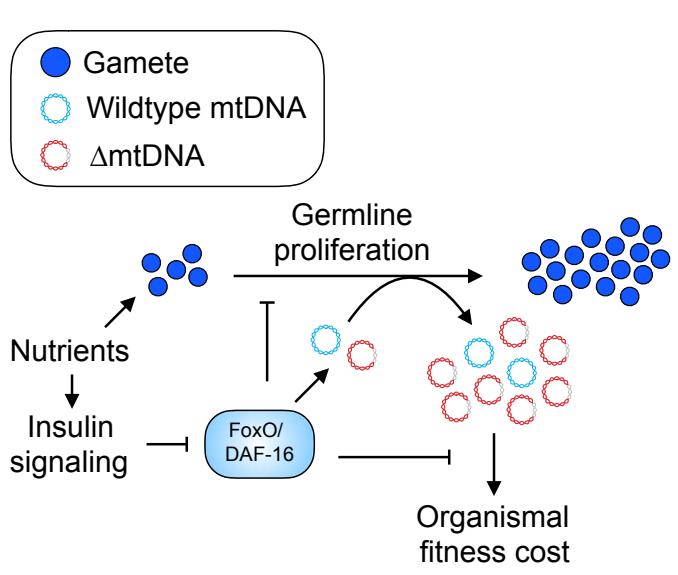


bioRxiv preprint doi: https://doi.org/10.1101/2020.01.30.927202; this version posted January 31, 2020. The copyright holder for this preprint (which was not certified by peer review) is the author/funder, who has granted bioRxiv a license to display the preprint in perpetuity. It is made available under aCC-BY-NC-ND 4.0 International license.

\section{Figure S1, related to Figure 1}

A

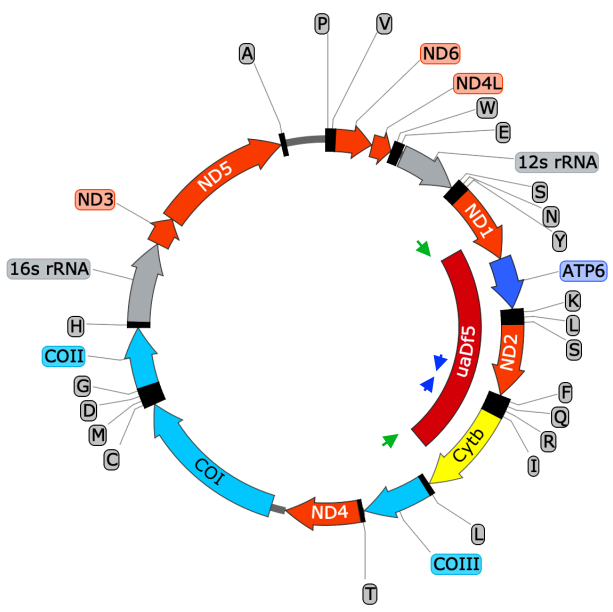

B

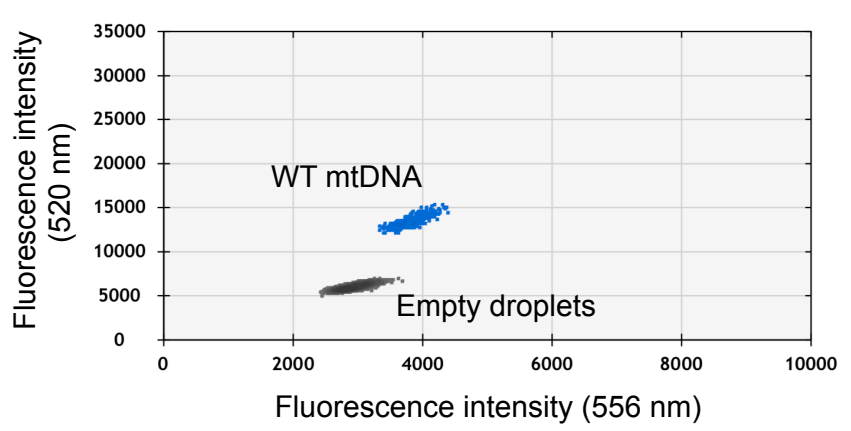

C

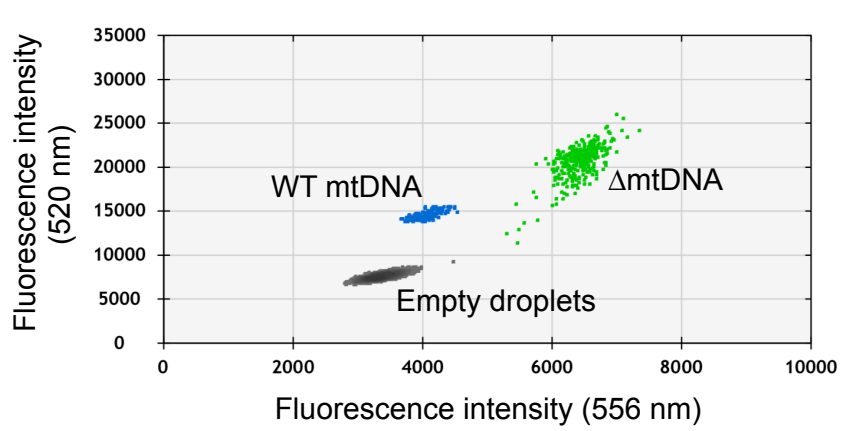


bioRxiv preprint doi: https://doi.org/10.1101/2020.01.30.927202; this version posted January 31, 2020. The copyright holder for this preprint (which was not certified by peer review) is the author/funder, who has granted bioRxiv a license to display the preprint in perpetuity. It is made available under aCC-BY-NC-ND 4.0 International license.

\section{Figure S2, related to Figure 2}

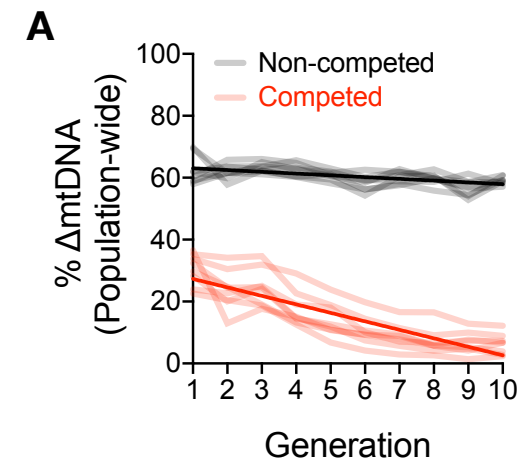

C
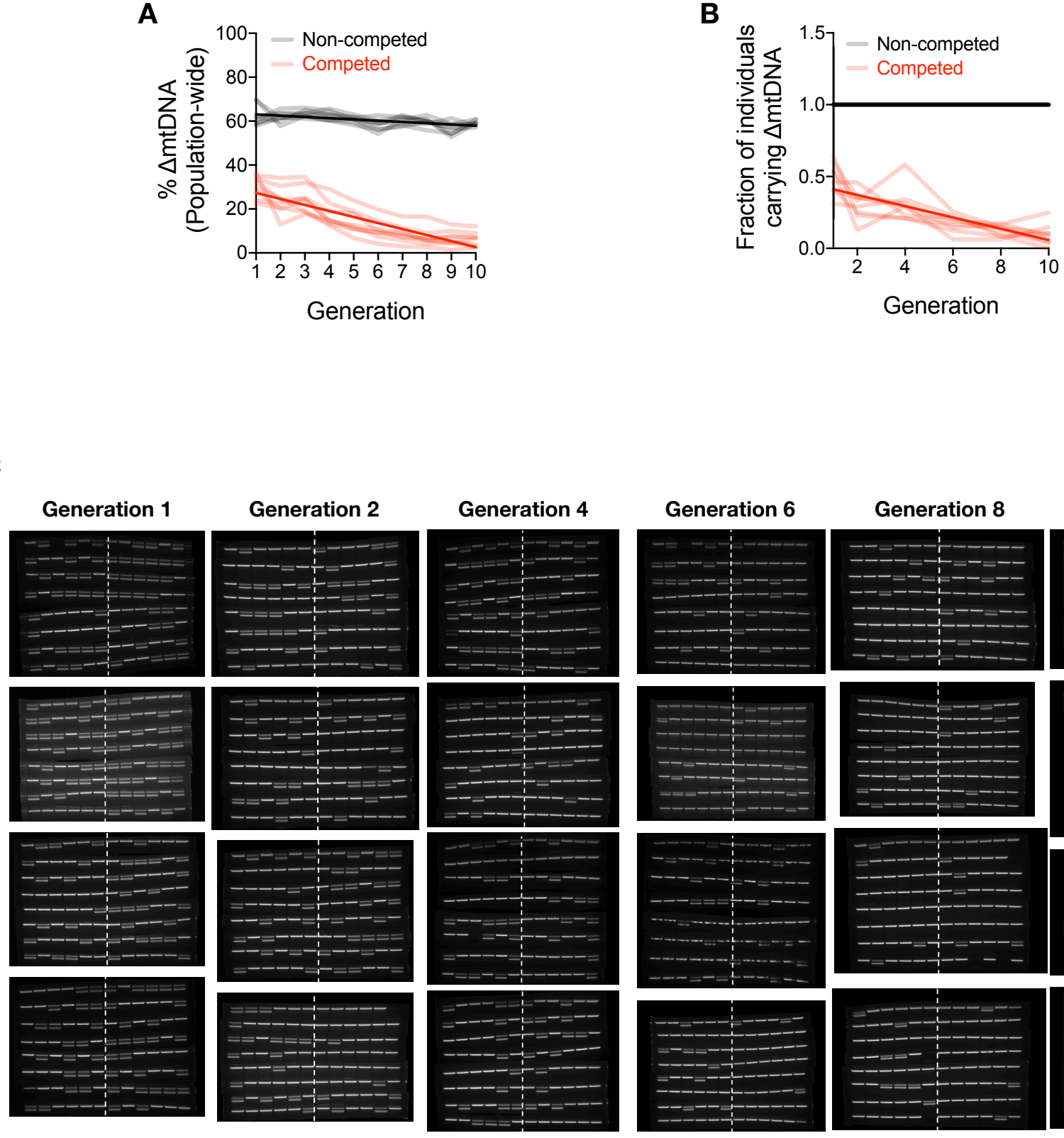

Generation 10
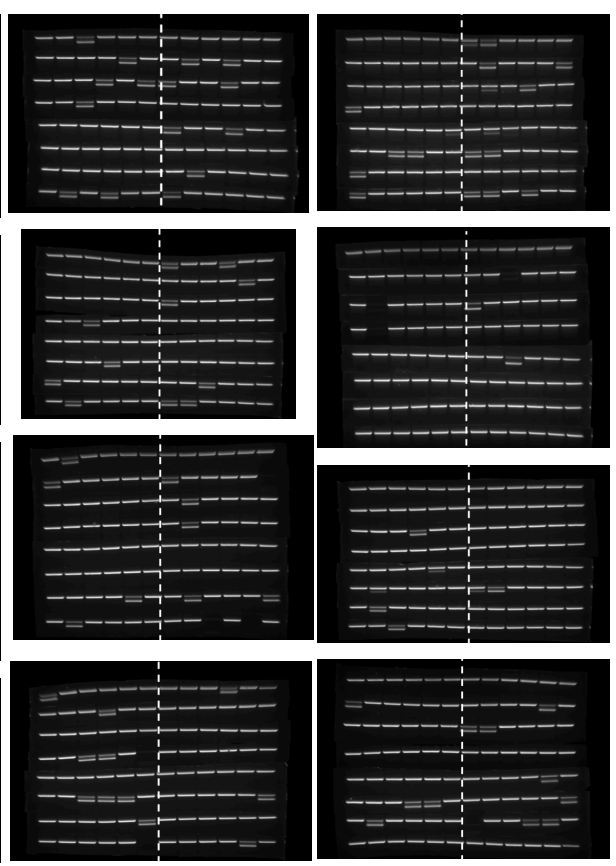
bioRxiv preprint doi: https://doi. org/10.1101/2020.01.30.927202; this version posted January 31, 2020. The copyright holder for this preprint (which was not certified by peer review) is the author/funder, who has granted bioRxiv a license to display the preprint in perpetuity. It is made available under aCC-BY-NC-ND 4.0 International license.

\section{Figure S3, related to Figure 3}

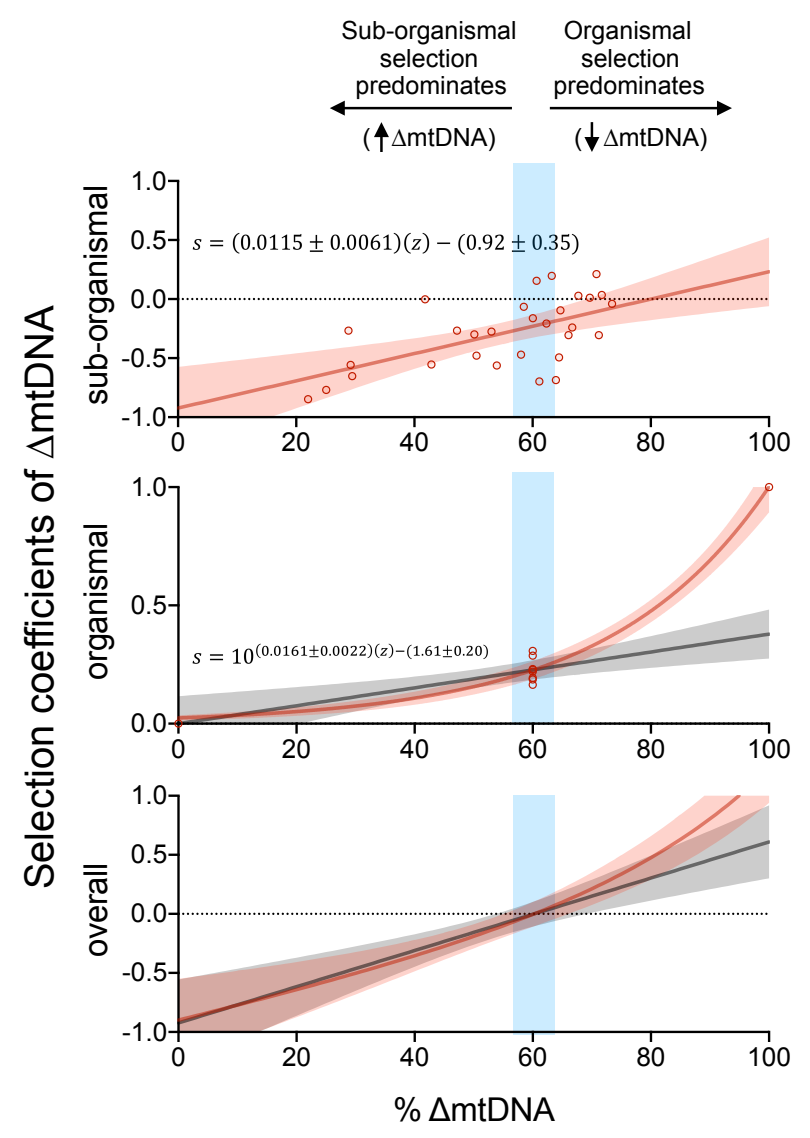


bioRxiv preprint doi: https://doi.org/10.1101/2020.01.30.927202; this version posted January 31, 2020. The copyright holder for this preprint (which was not certified by peer review) is the author/funder, who has granted bioRxiv a license to display the preprint in perpetuity. It is made available under aCC-BY-NC-ND 4.0 International license.

\section{Figure S4, related to Figure 3}

The Price Equation:

$w \Delta z=\operatorname{cov}(w, z)+E\left(w_{i}, \Delta z_{i}\right)$

Where:

$z=$ trait value in a population

$w=$ fitness of the trait in the population

$\Delta z=$ average change in trait value $z$

$w_{i}=$ fitness of individual unit $i$ within the population

$\Delta z_{i}=$ the change in trait value of individual $i$ between one generation and the next

$E=$ expected (average) value for the relationship between $\Delta z_{i}$ and $w_{i}$

Let the unit $i$ be defined as a molecule of mtDNA. The trait value $z_{i}$ can therefore be defined as the genotype of individual unit $i$, namely either $\triangle \mathrm{mtDNA}$ or wildtype mtDNA, and average trait value $z$ can be defined as the relative prevalence of $\triangle \mathrm{mtDNA}$ and wildtype mtDNA. The frequency of $\triangle \mathrm{mtDNA}$ is therefore a simple way to define overall trait value $z$ within a population. Fitness $w$ of $\triangle \mathrm{mtDNA}$ refers to the replication success of $\triangle \mathrm{mtDNA}$ relative to wildtype mtDNA. Selection coefficient $s$ is defined as $1-w$ and falls between 0 and 1 if $\triangle$ mtDNA is subject to negative selection. Conversely, if $\triangle \mathrm{mtDNA}$ is positively selected for, then wildtype mtDNA is subject to negative selection and $s_{w}$ falls between 0 and 1 , thus $s_{\triangle}$ falls between -1 and 0 , where $s_{w}$ and $s_{\triangle}$ are the selection coefficients for selection against wildtype mtDNA and $\triangle \mathrm{mtDNA}$, respectively. The selection coefficient for $\triangle \mathrm{mtDNA}$ therefore falls between -1 and 1 ( $s_{\Delta}<0$ indicates selection for $\triangle \mathrm{mtDNA}$ and $s_{\Delta}>0$ indicates selection against $\left.\triangle \mathrm{mtDNA}\right)$. Since replication is expected to preserve genotype ( $\triangle \mathrm{mtDNA}$ does not convert to wildtype upon replication, and vice versa), $\Delta z_{i}$ can be defined as zero, since the value of $z_{i}$ does not change between parent molecule and its replicate copies (Price, 1972). The remaining equation indicates that the average change in trait value across the population, $\Delta z$, is proportional to the covariance between trait value $z$ and fitness $w$, or as the covariance between $z$ and $s$ :

$$
\Delta z=\operatorname{cov}(s, z)
$$

Because $\triangle \mathrm{mtDNA}$ frequency covaries with the strength of selection acting on $\triangle \mathrm{mtDNA}$ at both organismal and suborganismal levels (regressions shown in Figure 3), the overall population-wide covariance between $\triangle \mathrm{mtDNA}$ selection coefficient $s$ and frequency $z$ can be defined as the sum of the sub-organismal and organismal covariances:

$$
\operatorname{cov}(s, z)_{\text {Total }}=\operatorname{cov}(s, z)_{\text {sub-organismal }}+\operatorname{cov}(s, z)_{\text {organismal }}
$$

Covariance defines the relationship between variation in a dataset along two axes or dimensions of measurement, for example trait value $z$ and selection coefficient $s$, as described by the following formula:

$$
\operatorname{cov}(s, z)=\sum_{k} \frac{\left(s_{k}-\bar{s}\right)\left(z_{k}-\bar{z}\right)}{n-1}
$$

Where:

$k=$ a collection of individual units $i$ (a group of organisms in the case of organismal selection; a single organism in the case of sub-organismal selection, with $i$ being defined the same as above)

$\bar{z}=$ average $z$ across all collections $k$ of individual units $i$

$\bar{s}=$ average $s$ across all collections $k$ of individual units $i$

Thus, the covariance can be used to describe the relationship between the distribution among values for trait $z$ and the distribution among corresponding values for selection coefficient $s$ :

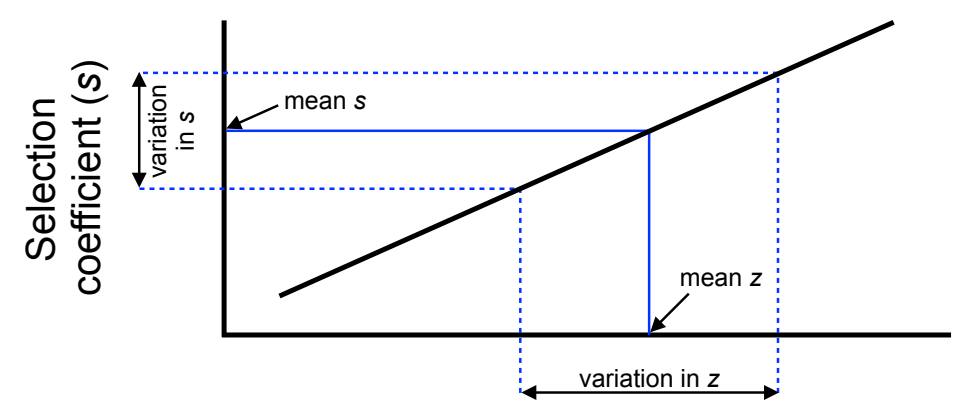

Trait ( $z)$ 
bioRxiv preprint doi: https://doi.org/10.1101/2020.01.30.927202; this version posted January 31, 2020. The copyright holder for this preprint (which was not certified by peer review) is the author/funder, who has granted bioRxiv a license to display the preprint in perpetuity. It is made available under aCC-BY-NC-ND 4.0 International license.

\section{Figure S5, related to Figure 4}

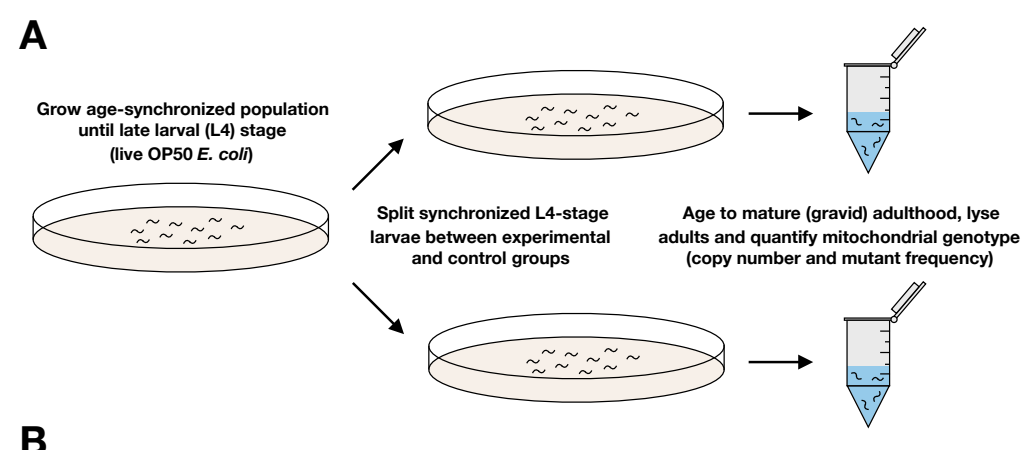

B

\begin{tabular}{|c|c|c|c|}
\hline Experiment & Food source & Experimental condition & Control \\
\hline Dietary restriction & $\begin{array}{l}\text { UV-inactivated OP50 } \\
\text { E. coli }\end{array}$ & $\begin{array}{l}\text { Control diet diluted } 100 \text {-fold in } \\
\text { M9 buffer }\end{array}$ & $\begin{array}{l}\text { OP50 E. coli resuspended in M9 } \\
\text { buffer and UV-inactivated }\end{array}$ \\
\hline $\begin{array}{l}\text { Insulin signaling } \\
\text { inactivation }\end{array}$ & $\begin{array}{l}\text { Live OP50 E. coli } \\
\text { or RNAi HT115 E. coli }\end{array}$ & $\begin{array}{l}25^{\circ} \mathrm{C} \text { with temperature-sensitive } \\
\text { daf-2(e 1370) or siRNA Aagainst } \\
\text { wildtype daf-2 }\end{array}$ & $\begin{array}{l}25^{\circ} \mathrm{C} \text { with wildtype daf-2 and live } \\
\text { OP50 E. colior control (siinNA-free) } \\
\text { HT115 E. coli }\end{array}$ \\
\hline $\begin{array}{l}\text { UV inactivation of } \\
\text { bacterial growth }\end{array}$ & $\begin{array}{l}\text { OP50 E. coli (live or } \\
\text { UV-inactivated) }\end{array}$ & UV-inactivated OP50 E. coli & Live OP50 E. coli \\
\hline
\end{tabular}
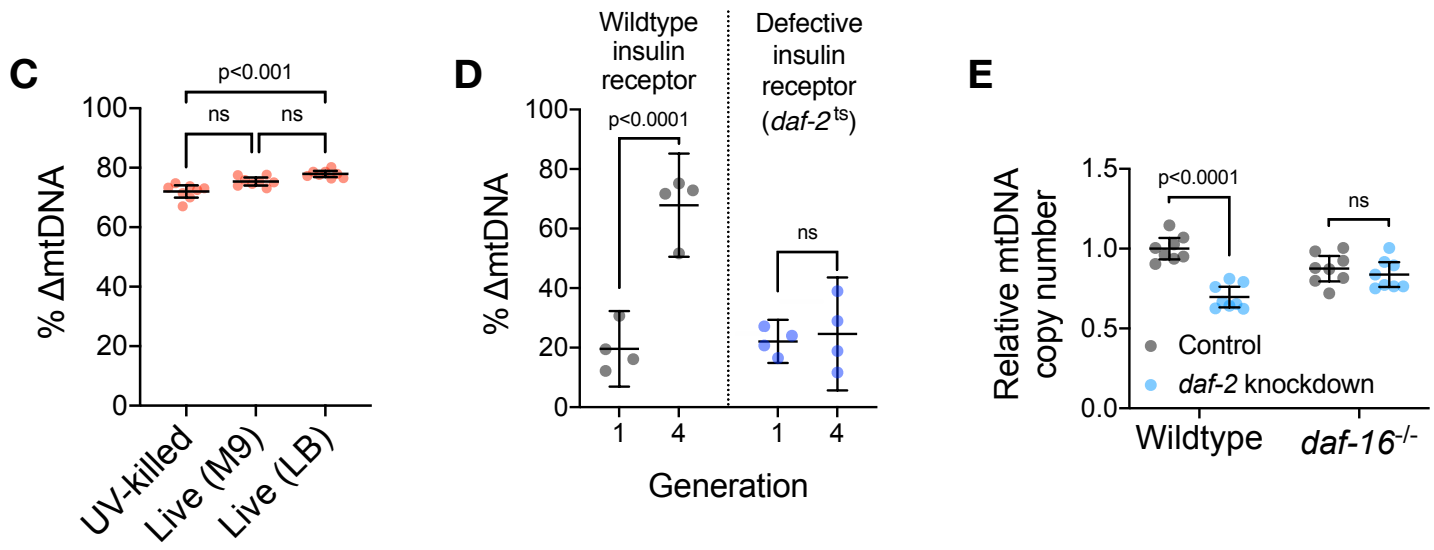

Dietary bacteria 
bioRxiv preprint doi: https://doi.org/10.1101/2020.01.30.927202; this version posted January 31, 2020. The copyright holder for this preprint (which was not certified by peer review) is the author/funder, who has granted bioRxiv a license to display the preprint in perpetuity. It is made available under aCC-BY-NC-ND 4.0 International license.

\section{Figure S6, related to Figure 5}

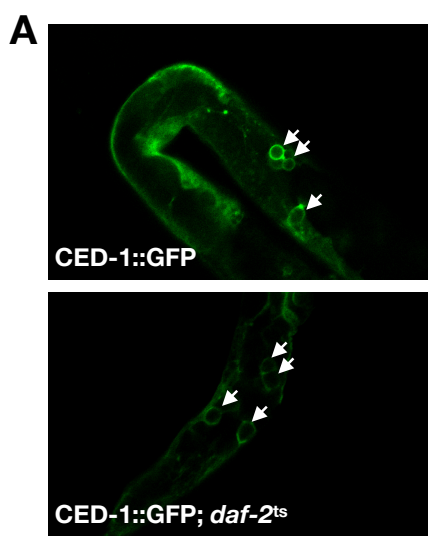

B

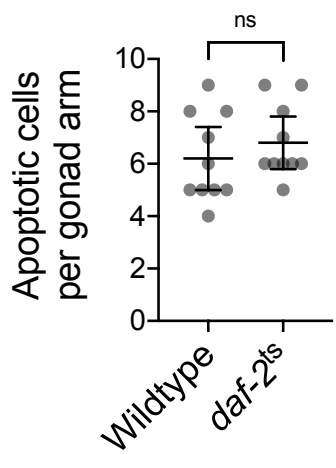


bioRxiv preprint doi: https://doi.org/10.1101/2020.0130.927202; this version posted January 31,2020. The copyright holder for this preprint (which was not certified by peer review) is the author/funder, who has granted bioRxiv a license to display the preprint in perpetuity. It is made available under aCC-BY-NC-ND 4.0 International license.

\section{Figure S7, related to Figure 7}

A

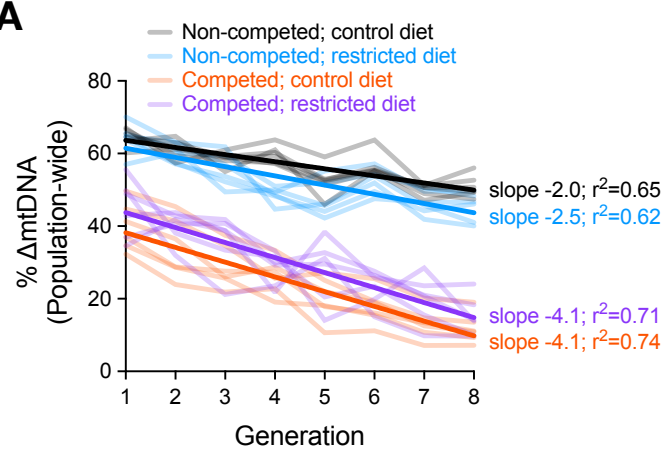

B

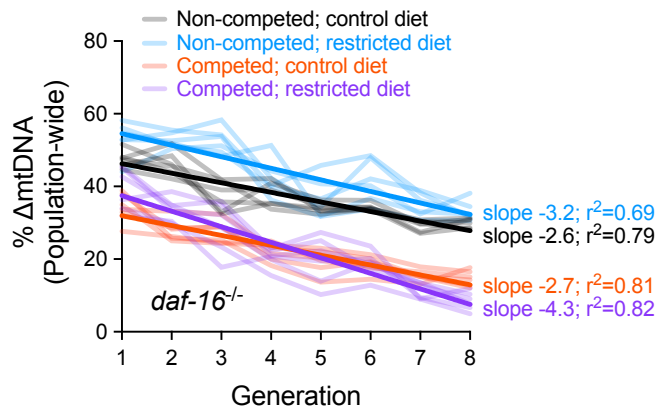

C

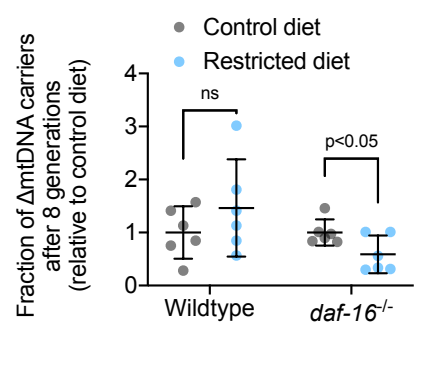

D

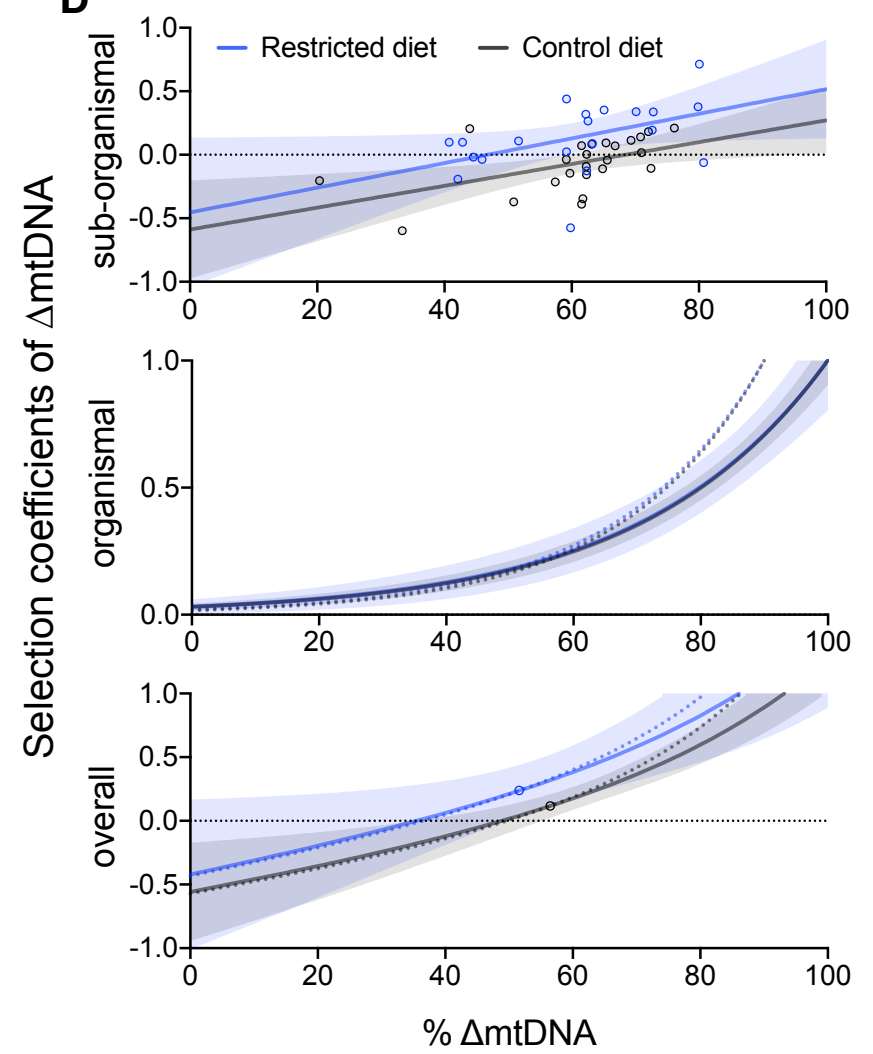

E
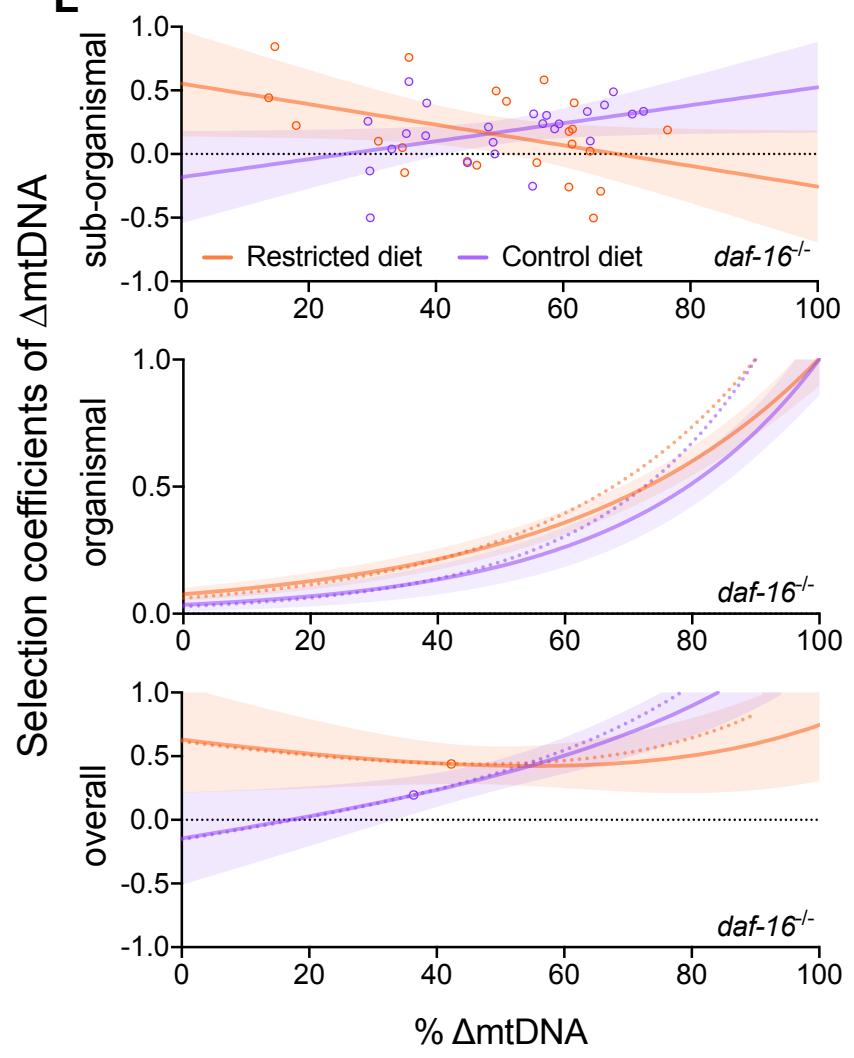
bioRxiv preprint doi: https://doi.org/10.1101/2020.01.30.927202; this version posted January 31, 2020. The copyright holder for this preprint (which was not certified by peer review) is the author/funder, who has granted bioRxiv a license to display the preprint in perpetuity. It is made available under aCC-BY-NC-ND 4.0 International license.

\section{Tables S1 and S2}

Table S1 - Raw shifts in $\triangle$ mtDNA at sub-organismal and organismal levels

\begin{tabular}{|l|c|c|} 
Units & Sub-organismal & Organismal \\
X-axis & $\% \Delta$ mtDNA (parents) & Time (generations) \\
Y-axis & $\begin{array}{c}\text { Shift in \% } \Delta \text { mtDNA } \\
\text { (progeny - parent) }\end{array}$ & $\begin{array}{c}\text { Population-wide \% } \Delta \text { mtDNA } \\
\text { (Mean individual \% } \Delta \text { mtDNA: 60) }\end{array}$ \\
Best-fit values & & -2.743 \\
Slope & -0.1975 & 30.07 \\
Intercept & 15.92 & \\
Std. Error & & 0.206 \\
Slope & 0.0546 & 1.278 \\
Intercept & 3.11 & \\
Goodness of Fit & & 0.6945 \\
R square & 0.3183 & 5.292 \\
Sy.X & 4.465 & \\
Linear regression statistics & & 177.3 \\
N & 30 & $<0.0001$ \\
F & 13.08 & \\
P (non-zero slope) & 0.0012 &
\end{tabular}

Table S2 - Selection coefficient at sub-organismal and organismal levels

\begin{tabular}{|l|c|c|} 
Selection level & Sub-organismal & Organismal \\
Units & & \\
X-axis & $\% \Delta$ mtDNA (parents) & Mean heteroplasmic \% $\Delta$ mtDNA \\
Y-axis & Selection coefficient & Selection coefficient \\
& & \\
Best-fit values & 0.0115 & 0.0161 \\
Slope & -0.92 & -1.61 \\
Intercept & & \\
Std. Error & 0.0030 & 0.0009 \\
Slope & 0.17 & 0.08 \\
Intercept & & \\
& & \\
Goodness of Fit & 0.35 & 0.97 \\
R square & 0.24 & 0.05 \\
Sy.X & & \\
Linear regression statistics & 30 & N/A \\
N & 14.9 & N/A \\
F & $<0.001$ & \\
P (non-zero slope) & &
\end{tabular}


bioRxiv preprint doi: https://doi.org/10.1101/2020.01.30.927202; this version posted January 31, 2020. The copyright holder for this preprint (which was not certified by peer review) is the author/funder, who has granted bioRxiv a license to display the preprint in perpetuity. It is made available under aCC-BY-NC-ND 4.0 International license.

\section{Tables S3 and S4}

Table S3 - Raw shifts in $\triangle$ mtDNA frequency by diet, daf-16 genotype, and parental $\Delta$ mtDNA level

\begin{tabular}{|c|c|c|c|c|}
\hline \multirow{2}{*}{$\begin{array}{l}\text { Genotype } \\
\text { Diet }\end{array}$} & \multicolumn{2}{|c|}{ Wildtype } & \multicolumn{2}{|c|}{ daf-16-- } \\
\hline & Control (ad libitum) & Restricted & Control (ad libitum) & Restricted \\
\hline \multicolumn{5}{|l|}{ Units } \\
\hline $\mathrm{X}$-axis & $\% \Delta$ mtDNA (parents) & $\% \triangle \mathrm{mtDNA}$ (parents) & $\% \Delta$ mtDNA (parents) & $\% \triangle \mathrm{mtDNA}$ (parents) \\
\hline Y-axis & $\begin{array}{l}\text { Shift in \% } \Delta \mathrm{mtDNA} \\
\text { (progeny - parent) }\end{array}$ & $\begin{array}{l}\text { Shift in \% } \Delta \mathrm{mtDNA} \\
\text { (progeny - parent) }\end{array}$ & $\begin{array}{l}\text { Shift in } \% \Delta \text { mtDNA } \\
\text { (progeny - parent) }\end{array}$ & $\begin{array}{l}\text { Shift in } \% \Delta \text { mtDNA } \\
\text { (progeny - parent) }\end{array}$ \\
\hline \multicolumn{5}{|l|}{ Best-fit values } \\
\hline Slope & -0.157 & -0.294 & -0.189 & 0.0978 \\
\hline Intercept & 10.66 & 13.34 & 4.301 & -9.319 \\
\hline \multicolumn{5}{|l|}{ Standard Error } \\
\hline Slope & 0.064 & 0.125 & 0.086 & 0.107 \\
\hline Intercept & 3.971 & 7.78 & 4.494 & 5.5 \\
\hline \multicolumn{5}{|l|}{ Goodness of Fit } \\
\hline R square & 0.214 & 0.234 & 0.181 & 0.0402 \\
\hline Sy.x & 3.889 & 7.063 & 5.772 & 8.734 \\
\hline \multicolumn{5}{|c|}{ Linear regression statistics } \\
\hline $\mathrm{N}$ & 24 & 20 & 24 & 22 \\
\hline $\mathrm{F}$ & 5.992 & 5.5 & 4.845 & 0.8378 \\
\hline $\mathrm{P}$ (non-zero slope) & 0.0228 & 0.0307 & 0.0385 & 0.3709 \\
\hline
\end{tabular}

Table S4 - Organismal selection on $\triangle$ mtDNA by diet and daf-16 genotype

\begin{tabular}{|l} 
Genotype \\
Diet \\
Units \\
X-axis \\
Y-axis \\
\\
Best-fit values \\
Slope \\
Intercept \\
Standard Error \\
Slope \\
Intercept \\
Goodness of Fit \\
R square \\
Sy.X \\
Linear regression statistics \\
N \\
F \\
P (non-zero slope) \\
P (effect of diet)
\end{tabular}

\begin{tabular}{|c|c|}
\hline \multicolumn{2}{|c|}{ Wildtype } \\
\hline Control (ad libitum) & Restricted \\
\hline Time (generations) & Time (generations) \\
\hline $\begin{array}{l}\text { Relative \% } \triangle \mathrm{mtDNA} \\
\text { (population / individual) }\end{array}$ & $\begin{array}{l}\text { Relative \% } \Delta \text { mtDNA } \\
\text { (population / individual) }\end{array}$ \\
\hline-0.0568 & -0.0515 \\
\hline 0.668 & 0.777 \\
\hline 0.0059 & 0.0076 \\
\hline 0.0298 & 0.0386 \\
\hline 0.668 & 0.497 \\
\hline 0.094 & 0.121 \\
\hline 48 & 48 \\
\hline 92.65 & 45.44 \\
\hline$<0.0001$ & $<0.0001$ \\
\hline
\end{tabular}

\begin{tabular}{|c|c|}
\hline \multicolumn{2}{|c|}{ daf-16-- } \\
\hline Control (ad libitum) & Restricted \\
\hline Time (generations) & Time (generations) \\
\hline $\begin{array}{l}\text { Relative \% } \triangle \mathrm{mtDNA} \\
\text { (population / individual) }\end{array}$ & $\begin{array}{c}\text { Relative \% } \Delta \text { mtDNA } \\
\text { (population / individual) }\end{array}$ \\
\hline-0.0306 & -0.0623 \\
\hline 0.733 & 0.776 \\
\hline 0.0052 & 0.0067 \\
\hline 0.0265 & 0.0336 \\
\hline 0.424 & 0.656 \\
\hline 0.083 & 0.106 \\
\hline 48 & 48 \\
\hline 33.88 & 87.55 \\
\hline$<0.0001$ & $<0.0001$ \\
\hline
\end{tabular}


bioRxiv preprint doi: https://doi.org/10.1101/2020.01.30.927202; this version posted January 31,2020. The copyright holder for this preprint (which was not certified by peer review) is the author/funder, who has granted bioRxiv a license to display the preprint in perpetuity. It is made available under aCC-BY-NC-ND 4.0 International license.

\section{Tables S5 and S6}

Table S5 - Sub-organismal selection coefficients by diet, daf-16 genotype, and parental $\triangle$ mtDNA level

\begin{tabular}{|c|c|c|c|c|}
\hline \multirow{2}{*}{$\begin{array}{l}\text { Genotype } \\
\text { Diet }\end{array}$} & \multicolumn{2}{|c|}{ Wildtype } & \multicolumn{2}{|c|}{ daf-16-/- } \\
\hline & Control (ad libitum) & Restricted & Control (ad libitum) & Restricted \\
\hline \multicolumn{5}{|l|}{ Units } \\
\hline $\mathrm{X}$-axis & $\% \Delta$ mtDNA (parents) & $\% \Delta$ mtDNA (parents) & $\% \Delta$ mtDNA (parents) & $\% \Delta$ mtDNA (parents) \\
\hline Y-axis & Selection coefficient & Selection coefficient & Selection coefficient & Selection coefficient \\
\hline \multicolumn{5}{|l|}{ Best-fit values } \\
\hline Slope & 0.0086 & 0.0097 & 0.0071 & -0.0081 \\
\hline Intercept & -0.59 & -0.45 & -0.18 & 0.55 \\
\hline \multicolumn{5}{|l|}{ Standard Error } \\
\hline Slope & 0.0030 & 0.0045 & 0.0033 & 0.0039 \\
\hline Intercept & 0.19 & 0.28 & 0.17 & 0.20 \\
\hline \multicolumn{5}{|l|}{ Goodness of Fit } \\
\hline R square & 0.27 & 0.21 & 0.17 & 0.18 \\
\hline Sy.x & 0.18 & 0.25 & 0.22 & 0.32 \\
\hline \multicolumn{5}{|c|}{ Linear regression statistics } \\
\hline $\mathrm{N}$ & 24 & 20 & 24 & 20 \\
\hline $\mathrm{F}$ & 8.16 & 4.66 & 4.48 & 4.36 \\
\hline P (non-zero slope) & $<0.01$ & $<0.05$ & $<0.05$ & $<0.05$ \\
\hline
\end{tabular}

Table S6 - Organismal selection coefficients by diet, daf-16 genotype, and $\triangle \mathrm{mtDNA}$ level

\begin{tabular}{|l} 
Genotype \\
Diet \\
Units \\
X-axis \\
Y-axis \\
Best-fit values \\
Slope \\
Intercept \\
Standard Error \\
Slope \\
Intercept \\
Goodness of Fit \\
R square \\
Sy.X
\end{tabular}

$\begin{array}{|cc|}\text { Control (ad libitum) } & \text { Wildtype } \\ \text { Mean heteroplasmic } \% \Delta \text { mtDNA } & \text { Restricted diet } \\ & \text { Mean heteroplasmic } \% \Delta \text { mtDNA } \\ \text { Selection coefficient } & \text { Selection coefficient } \\ & \\ 0.0151 & 0.0149 \\ -1.51 & -1.49 \\ & \\ 0.0008 & 0.0017 \\ 0.08 & 0.16 \\ 0.98 & \\ 0.04 & 0.94 \\ & 0.08\end{array}$

\begin{tabular}{|c|c|}
\hline \multicolumn{2}{|c|}{ daf-16-/- } \\
\hline Control (ad libitum) & Restricted diet \\
\hline Mean heteroplasmic $\% \Delta$ mtDNA & Mean heteroplasmic $\% \Delta \mathrm{mtDNA}$ \\
\hline Selection coefficient & Selection coefficient \\
\hline 0.0145 & 0.0112 \\
\hline-1.45 & -1.115 \\
\hline 0.0014 & 0.0007 \\
\hline 0.13 & 0.06 \\
\hline 0.97 & 0.98 \\
\hline 0.06 & 0.04 \\
\hline
\end{tabular}

